\title{
Liquid Polyamorphism: Some Unsolved Puzzles of Water in Bulk, Nanoconfined, and Biological Environments
}

\author{
H. E. Stanley*, Pradeep Kumar*, Giancarlo Franzese ${ }^{\dagger}$, Limei Xu*, Zhenyu Yan*, \\ Marco G. Mazza*, S.-H. Chen**, F. Mallamace*, and S. V. Buldyrev ${ }^{\S *}$ \\ ${ }^{*}$ Center for Polymer Studies and Dept. of Physics, Boston University, Boston, MA 02215 USA \\ ${ }^{\dagger}$ Departament de Física Fonamental, Univ. de Barcelona, Diagonal 647, Barcelona 08028, SPAIN \\ **Nuclear Science and Engineering Dept., Mass. Inst. of Technology, Cambridge, MA 02139 USA \\ ¥Dipartimento di Fisica, Univ. Messina, Vill. S. Agata, C.P. 55, I-98160 Messina, ITALY

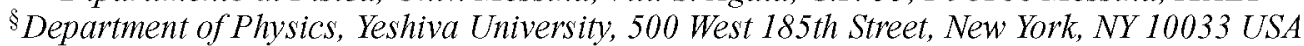

\begin{abstract}
We investigate the relation between changes in dynamic and thermodynamic anomalies arising from the presence of the liquid-liquid critical point in (i) Two models of water, TIP5P and ST2, which display a first order liquid-liquid phase transition at low temperatures; (ii) the Jagla model, a spherically symmetric two-scale potential known to possess a liquid-liquid critical point, in which the competition between two liquid structures is generated by repulsive and attractive ramp interactions; and (iii) A Hamiltonian model of water where the idea of two length/energy scales is built in; this model also displays a first order liquid-liquid phase transition at low temperatures besides the first order liquid-gas phase transition at high temperatures. We find a correlation between the dynamic fragility crossover and the locus of specific heat maxima $C_{P}^{\max }$ ("Widom line") emanating from the critical point. Our findings are consistent with a possible relation between the previously hypothesized liquid-liquid phase transition and the transition in the dynamics recently observed in neutron seattering experiments on confined water. More generally, we argue that this connection between $C_{P}^{\max }$ and the dynamic crossover is not limited to the case of water, a hydrogen bonded network liquid, but is a more general feature of crossing the Widom line, an extension of the first-order coexistence line in the supercritical region. We present evidence from experiments and computer simulations supporting the hypothesis that water displays polyamorphism, i.e., water separates into two distinct liquid phases. This concept of a new liquid-liquid phase transition is finding application to other liquids as well as water, such as silicon and silica. We also discuss related puzzles, such as the mysterious behavior of confined water and the "skin" of hydration water near a biomolecule. Specifically, using molecular dynamics simulations, we also investigate the relation between the dynamic transitions of biomolecules (lysozyme and DNA) and the dynamic and thermodynamic properties of hydration water. We find that the dynamic transition of the macromolecules, sometimes called a "protein glass transition", occurs at the temperature of dynamic crossover in the diffusivity of hydration water, and also coincides with the maxima of the isobaric specific heat $C_{P}$ and the temperature derivative of the orientational order parameter. We relate these findings to the hypothesis of a liquid-liquid critical point in water. Our simulations are consistent with the possibility that the protein glass transition results from a change in the behavior of hydration water, specifically from crossing the Widom line.
\end{abstract}

Keywords: High-density liquid, Liquid-liquid critical point, Liquid-liquid phase transition, Low-density liquid PACS: 61.20.Ja, 61.20.Gy

\section{Background}

One "mysterious" property of liquid water was recognized 300 years ago [1]: although most liquids contract as temperature decreases, liquid bulk water begins to expand when its temperature drops below $4^{\circ} \mathrm{C}$. Indeed, a simple kitchen experiment demonstrates that the bottom layer of a glass of unstirred iced water remains at $4^{\circ} \mathrm{C}$ while colder layers of $0^{\circ} \mathrm{C}$ water "float" on top (cf., Fig. 1 of Ref. [2]). The mysterious properties of liquid bulk water become more pronounced in the supercooled region below $0^{\circ} \mathrm{C}[3,4,5]$. For example, if the coefficient of thermal expansion $\alpha_{P}$, isothermal compressibility $K_{T}$, and constant-pressure specific heat $C_{P}$ are extrapolated below the lowest temperatures measurable they would become infinite at a temperature of $T_{s} \approx 228 \mathrm{~K}[3,6]$.
Water is a liquid, but glassy water-also called amorphous ice-can exist when the temperature drops below the glass transition temperature $T_{g}$. Although it is a solid, its structure exhibits a disordered molecular liquidlike arrangement. Low-density amorphous ice (LDA) has been known for 60 years [7], and a second kind of amorphous ice, high-density amorphous ice (HDA) was discovered in $1984[8,9,10]$. HDA has a structure similar to that of high-pressure liquid water, suggesting that HDA may be a glassy form of high-pressure water [11, 12], just as LDA may be a glassy form of low-pressure water. Water has at least two different amorphous solid forms, a phenomenon called polyamorphism $[13,14,15,16,17$, $18,19]$, and recently additional forms of glassy water have been the focus of active experimental and computational investigation $[20,21,22,23,24,25,26,27,28]$.

\footnotetext{
CP982, Complex Systems, $5^{\text {th }}$ International Workshop on Complex Systems

edited by M. Tokuyama, I. Oppenheim, and H. Nishiyama
}

(C) 2008 American Institute of Physics 978-0-7354-0501-1/08/\$23.00 


\section{Current Hypotheses}

Many classic "explanations" for the mysterious behavior of liquid bulk water have been developed [29, 30, 31, $32,33,34]$, including a simple two-state model dating back to Röntgen [35] and a clathrate model dating back to Pauling [36]. Three hypotheses are under current discussion:

(i) The stability limit hypothesis [37], which assumes that the spinodal temperature line $T_{S}(P)$ between two liquids with different densities in the pressuretemperature $(P-T)$ phase diagram connects at negative $P$ to the locus of the liquid-to-gas spinodal for superheated bulk water. Liquid water cannot exist when cooled or stretched beyond the line $T_{S}(P)$.

(ii) The singularity-free hypothesis [38], considers the possibility that the observed polyamorphic changes in water resemble a genuine transition, but is not. For example, if water is a locally-structured transient gel comprised of molecules held together by hydrogen bonds whose number increases as temperature decreases [39, 40,41], then the local "patches" or bonded sub-domains $[42,43]$ lead to enhanced fluctuations of specific volume and entropy and negative cross-correlations of volume and entropy whose anomalies closely match those observed experimentally.

(iii) The liquid-liquid (LL) phase transition hypothesis [44] arose from MD studies on the structure and equation of state of supercooled bulk water and has received some support $[45,46,47,48,49,50$, $51,52,53]$. Below the hypothesized second critical point the liquid phase separates into two distinct liquid phases: a low-density liquid (LDL) phase at low pressures and a high-density liquid (HDL) at high pressure (Fig. 1). Bulk water near the known critical point at $647 \mathrm{~K}$ is a fluctuating mixture of molecules whose local structures resemble the liquid and gas phases. Similarly, bulk water near the hypothesized LL critical point is a fluctuating mixture of molecules whose local structures resemble the two phases, LDL and HDL. These enhanced fluctuations influence the properties of liquid bulk water, thereby leading to anomalous behavior.

\section{Selected Experimental Results}

Many precise experiments have been performed to test the various hypotheses discussed in the previous section, but there is as yet no widespread agreement on which physical picture - if any - is correct. The connection between liquid water and the two amorphous ices predicted by the LL phase transition hypothesis is difficult to prove experimentally because supercooled water freezes spontaneously below the homogeneous nucleation temperature $T_{H}$, and amorphous ice crystallizes above the crystallization temperature $T_{X}[54,55,56]$. Crystallization makes experimentation on the supercooled liquid state between $T_{H}$ and $T_{X}$ almost impossible. However, comparing experimental data on amorphous ice at low temperatures with that of liquid water at higher temperatures allows an indirect discussion of the relationship between the liquid and amorphous states. It is found from neutron diffraction studies [12] and simulations that the structure of liquid water changes toward the LDA structure when the liquid is cooled at low pressures and changes toward the HDA structure when cooled at high pressures, which is consistent with the LL phase transition hypothesis [12]. The amorphous states (LDA and HDA) are presently considered to be "smoothly" connected thermodynamically to the liquid state if the entropies of the amorphous states are small $[57,58]$, and experimental results suggest that their entropies are indeed small [59].

In principle, it is possible to investigate experimentally the liquid state in the region between $T_{H}$ and $T_{X}$ during the extremely short time interval before the liquid freezes to crystalline ice $[17,56,60]$. Because high-temperature liquid bulk water becomes LDA without crystallization when it is cooled rapidly at one bar $[16,61]$, LDA appears directly related to liquid water. A possible connection between liquid bulk water at high pressure and HDA can be seen when ice crystals are melted using pressure [17]. Other experimental results [56] on the highpressure ices $[34,62]$ that might demonstrate a LL firstorder transition in the region between $T_{H}$ and $T_{X}$ have been obtained.

\section{Selected Results from Simulations}

Water is challenging to simulate because it is a molecular liquid and there is presently no precise yet tractable intermolecular potential that is universally agreed on. Nevertheless there are some distinct advantages of simulations over experiments. Experiments cannot probe the "No-Man's land" that arises in bulk water from homogeneous nucleation phenomena, but simulations have the advantage that they can probe the structure and dynamics well below $T_{H}$ since nucleation does not occur on the time scale of computer simulations. Of the three hypotheses above, the LL phase transition hypothesis is best supported by simulations, some using the ST2 potential which exaggerates the real properties of bulk water, and others using the SPC/E and TIP4P potentials which underestimate them $[44,63,64,65,66,67]$. Recently, simulations have begun to appear using the more reliable TIP5P potential $[68,69,70]$. The precise loca- 
tion of the LL critical point is difficult to obtain since the continuation of the first order line is a locus of maximum compressibility $[63,64,66]$.

Further, computer simulations may be used to probe the local structure of water. At low temperatures, many water molecules appear to possess one of two principal local structures, one resembling LDA and the other HDA $[63,44,65,71]$. Experimental data can also be interpreted in terms of two distinct local structures [72, 73, 74].

\section{Understanding "Static Heterogeneities"}

The systems in which water is confined are diverseincluding the rapidly-developing field of artificial "nanofluidic" systems (man-made devices of order of nanometer or less that convey fluids). Among the special reasons for our interest in confined water is that phenomena occurring at a given set of conditions in bulk water occur under perturbed conditions for confined water $[75,76,77,78,79,80,81,82,83,84,85,86,87,88]$. For example, the coordinates of the hypothesized LL critical point lie in the experimentally-inaccessible NoMan's land of the bulk water phase digram, but appear to lie in an accessible region of the phase diagrams of both two-dimensionally and one-dimensionally confined water $[89,90]$. Simulations have been carried out to understand the effect of purely geometrical confinement $[91,92,93,94,95,96]$ and of the interaction with hydrophilic $[97,98,99,100,101]$ or hydrophobic $[102,103,104,105]$ surfaces. It is interesting also to study the effects that confinement may have on the phase transition properties of supercooled water [96], in order to clarify the possible presence of a LL phase transition in the water. Recent work on the phase behavior of confined water suggests a sensitive dependence on the interaction with the surfaces [104], as a LL phase transition appears to be consistent with simulations of water confined between two parallel flat hydrophobic walls [94]. Works are in progress to extend this work to hydrophilic pores, such as those in Vycor glasses or biological situations, and to hydrophobic hydrogels, systems of current experimental interest $[106,107,108,109,110,111,112,113,114,115,94$, $116,117,118,119,120,121]$.

\section{Potentials with Two Characteristic Length Scales: Physical Arguments}

A critical point appears if the pair potential between two particles of the system exhibits a minimum, and Fig. 1a sketches the potential of such an idealized sys-

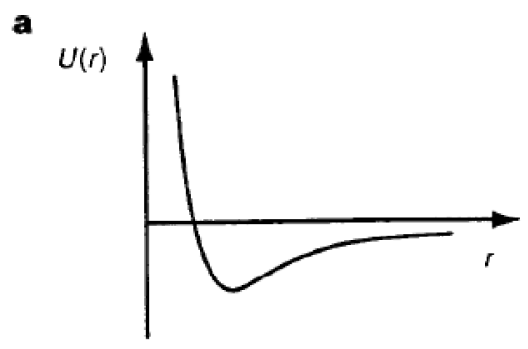

b
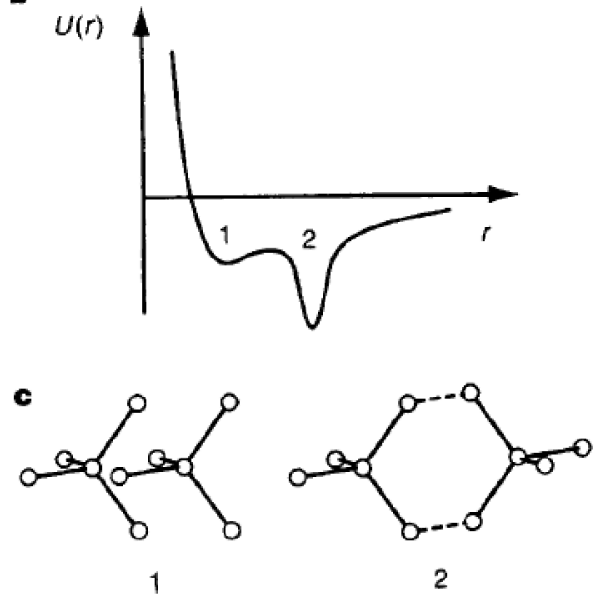

FIGURE 1. (a) Idealized system characterized by a pair interaction potential with a single attractive well. At low enough $T\left(T<T_{C}\right)$ and high enough $P\left(P>P_{C}\right)$, the system condenses into the "liquid" well shown. (b) Idealized system characterized by a pair interaction potential whose attractive well has two sub-wells, the outer of which is deeper and narrower. For low enough $T\left(T<T_{C^{\prime}}\right)$ and low enough $P\left(P<P_{C^{\prime}}\right)$, the onephase liquid can "condense" into the narrow outer "LDL" subwell, thereby giving rise to a LDL phase, and leaving behind the high-density liquid phase occupying predominantly the inner subwell. (c) Two idealized interaction clusters of water molecules in configurations that may correspond to the two sub-wells of (b).

tem. At high temperature, the system's kinetic energy is so large that the potential well does not have an effect, and the system is in a single "fluid" (or gas) phase. At low enough temperature $\left(T<T_{C}\right)$ and large enough pressure $\left(P>P_{C}\right)$ the fluid is sufficiently influenced by the minimum in the pair potential that it can condense into the low specific volume liquid phase. At lower pressure $\left(P<P_{C}\right)$, the system explores the full range of distances - the large specific volume gas phase.

If the potential well has the form shown in Fig. 1b the attractive potential well of Fig. 1a has now bifurcated into a deeper outer sub-well and a more shallow inner sub-well. Such a two-minimum ("two length scale") potential can give rise to the occurrence at low temperatures of a LL critical point at $\left(T_{C^{\prime}}, P_{C^{\prime}}\right)$ [122]. At high temperature, the system's kinetic energy is so large that 
the two sub-wells have no appreciable effect on the thermodynamics and the liquid phase can sample both subwells. However, at low enough temperature $\left(T<T_{C^{\prime}}\right)$ and not too high a pressure $\left(P<P_{C^{\prime}}\right)$ the system must respect the depth of the outer sub-well so the liquid phase "condenses" into the outer sub-well (the LDL phase). At higher pressure it is forced into the shallower inner subwell (the HDL phase).

The above arguments concern the average or "thermodynamic" properties, but they may also be useful in anticipating the local properties in the neighborhood of individual molecules [123]. Consider, again, an idealized fluid with a potential of the form of Fig. 1a. and suppose that $T$ is, say, $1.2 T_{C}$ so that the macroscopic liquid phase has not yet condensed out. Although the system is not entirely in the liquid state, small clusters of molecules begin to coalesce into the potential well, thereby changing their characteristic interparticle spacing (and hence their local specific volume) and their local entropy, so the fluid system will experience spatial fluctuations characteristic of the liquid phase even though this phase has not yet condensed out of the fluid at $T=1.2 T_{C}$. Specific volume fluctuations are measured by the isothermal compressibility and entropy fluctuations by the constant-pressure specific heat, so these two functions should start to increase from the values they would have if there were no potential well at all. As $T$ decreases toward $T_{C}$, the magnitude of the fluctuations (and hence of the compressibility and the specific heat) increases monotonically and in fact diverges to infinity as $T \rightarrow T_{C}$. The cross-fluctuations of specific volume and entropy are proportional to the coefficient of thermal expansion, and this (positive) function should increase without limit as $T \rightarrow T_{C}$.

Consider an idealized fluid with a potential of the form of Fig. 1b, and suppose that $T$ is now below $T_{C}$ but is 20 percent above $T_{C^{\prime}}$, so that the LDL phase has not yet condensed out. The liquid can nonetheless begin to sample the two sub-wells and clusters of molecules will begin to coalesce in each well, with the result that the liquid will experience spatial fluctuations characteristic of the LDL and HDL phase even though the liquid has not yet phase separated. The specific volume fluctuations and entropy fluctuations will increase, and so the isothermal compressibility $K_{T}$ and constant-pressure specific heat $C_{P}$ begin to diverge. Moreover, if the outer well is narrow, then when a cluster of neighboring particles samples the outer well it has a larger specific volume and a smaller entropy, so the anticorrelated cross-fluctuations of specific volume (the isothermal expansion coefficient $\alpha_{P}$ ) is now negative, and approaching $-\infty$ as $\mathrm{T}$ decreases toward $T_{C^{\prime}}$.

Now if by chance the value of $T_{C^{\prime}}$ is lower than the value of $T_{H}$, then the phase separation discussed above would occur only at temperatures so low that the liquid would have frozen! In this case, the "hint" of the LL crit- ical point $C^{\prime}$ is the presence of these local fluctuations whose magnitude would grow as $T$ decreases, but which would never actually diverge if the point $C^{\prime}$ is never actually reached. Functions would be observed experimentally to increase as if they would diverge to $\infty$ or $-\infty$ but at a temperature below the range of experimental accessibility.

Now consider not the above simplified potential, but rather the complex (and unknown) potential between nonlinear water molecules. The tetrahedrality of water dictates that the outermost well corresponds to the ordered configuration with lower entropy. Thus although we do not know the actual form of the intermolecular potential in bulk water, it is not implausible that the same considerations apply as those discussed for the simplified potential of Fig. 1b. Indeed, extensive studies of such pair potentials have been carried out recently and the existence of the LL critical point has been demonstrated in such models $[124,47,48,125,50,51,52,53,143,144$, $145,146,147]$.

To make more concrete how plausible it is to obtain a bifurcated potential well of the form of Fig. $1 \mathrm{~b}$, consider that one can crudely approximate water as a collection of 5-molecule groups called Walrafen pentamers (Fig. 1c) [73]. The interaction strengths of two adjacent Walrafen pentamers depends on their relative orientations. The first and the second energy minima of Fig. $1 \mathrm{~b}$ correspond to the two configurations of adjacent Walrafen pentamers with different mutual orientations (Fig. 1c).

The two local configurations $\# 1$ and $\# 2$ in Fig. $1 \mathrm{c}$ are (i) a high-energy, low specific volume, high-entropy, non-bonded \#1-state, or (ii) a low-energy, high specific volume, low-entropy, bonded \#2-state. The difference in local structure is also the difference in the local structure between a high-pressure crystalline ice (such as ice VI or ice VII) and a low-pressure crystalline ice (such as ice $\mathrm{I}_{h}$ ) [34] (Fig. 1c).

The region of the P-T plane along the line continuing from the LDL-HDL coexistence line extrapolated to higher temperatures above the second critical point is the locus of points where the LDL on the low-pressure side and the HDL on the high-pressure side are continuously transforming - it is called the Widom line, defined to be the locus of points where the correlation length is maximum. Near this line, two different kinds of local structures, having either LDL or HDL properties, "coexist" $[71,126,127]$. The entropy fluctuations are largest near the Widom line, so $C_{P}$ increases to a maximum, displaying a $\lambda$-like appearance [128]. The increase in $C_{P}$ [58] resembles the signature of a glass transition as suggested by mode-coupling theory $[129,130,131]$. Careful measurements and simulations of static and dynamic correlation functions $[126,132,133,134,135]$ may be useful in determining the exact nature of the apparent singular behavior near $220 \mathrm{~K}$. 


\section{Potentials with Two Characteristic Length Scales: Tractable Models}

The above discussion is consistent with the possible existence of two well-defined classes of liquids: simple and water-like. The former interact via sphericallysymmetric non-softened potentials, do not exhibit thermodynamic nor dynamic anomalies. We can calculate translational and orientational order parameters $(t$ and $q$ ), and project equilibrium state points onto the $(t, q)$ plane thereby generating what is termed the ErringtonDebenedetti (ED) order map [136, 43]. In water-like liquids, interactions are orientation-dependent; these liquids exhibit dynamic and thermodynamic anomalies, and their ED "order map" is in general two-dimensional but becomes linear (or quasi-linear) when the liquid exhibits structural, dynamic or thermodynamic anomalies.

Hemmer and Stell [137] showed that in fluids interacting via pairwise-additive, spherically-symmetric potentials consisting of a hard core plus an attractive tail, softening of the repulsive core can produce additional phase transitions. This pioneering study elicited a considerable body of work on so-called core-softened potentials which can generate water-like density and diffusion anomalies $[137,138,139,140,141,142,143,144$, $145,146,147,151,152,153,154,155,156]$. This important finding implies that strong orientational interactions, such as those that exist in water and silica, are not a necessary condition for a liquid to have thermodynamic and dynamic anomalies.

A softened-core potential has been used [122] to explain the isostructural solid-solid critical point present in materials such as $\mathrm{Cs}$ and $\mathrm{Ce}$, for which the shape of the effective pair potential obtained from scattering experiments is "core-softened" [4, 122, 140, 157, 158]. Analytical work in $1 \mathrm{D}$ suggests a LL phase transition, and the existence at $T=0$ of low and high density phases. Recent work using large-scale MD simulations reports anomalous behavior in $2 \mathrm{D}$ as well $[140,142]$. In $3 \mathrm{D}$ we showed that a squared potential with a repulsive shoulder and an attractive well displays a phase diagram with a LL critical point and no density anomaly $[143,144,145,146]$. The continuous version of the the same shouldered attractive potential shows not only the LL critical point, but also the density anomaly $[147,156]$. We use the soft-core potential to investigate the relationship between configurational entropy $S_{\text {conf }}$ and diffusion coefficient $D$. Recent work using the SPC/E potential [159] suggests that the temperature-density dependence of $S_{\text {conf }}$ may correlate with $D$, and that the maximum of $S_{\text {conf }}$ tracks the density maxima line.

Two questions arise naturally from this emerging taxonomy of liquid behavior. First, is structural order in core-softened fluids hard-sphere or water-like? Second, is it possible to seamlessly connect the range of liquid behavior from hard spheres to water-like by a simple and common potential, simply by changing a physical parameter?

In recent work, Yan et al. $[160,161,162]$ used a simple spherically-symmetric "hard-core plus ramp" potential to address the first question. They found that this coresoftened potential with two characteristic length scales not only gives rise to water-like diffusive and density anomalies, but also to an ED water-like order map, implying that orientational interactions are not necessary in order for a liquid to have structural anomalies. We are investigating the evolution of dynamic, thermodynamic and structural anomalies, using the ratio $\lambda$ of hard core and soft core length scales as a control parameter. We hope to show that the family of tunable sphericallysymmetric potentials so generated evolves continuously between hard sphere and water-like behavior; if successful, this will be the first demonstration that essential aspects of the wide range of liquid behavior encompassed by hard spheres and tetrahedrally-coordinated networkformers can be systematically traversed by varying a single control parameter. We will study the equation of state, diffusion coefficient, and structural order parameters $t$ and $q$. Our calculations seem to reveal a negative thermal expansion coefficient (static anomaly) and an increase of the diffusion coefficient upon isothermal compression (dynamic anomaly) for $0 \leq \lambda<6 / 7$. As in bulk water, the regions where these anomalies occur are nested domes in the $(T, \rho)$ or $(T, P)$ planes, with the "thermodynamic anomaly dome" contained within the "dynamic anomaly dome." The ED order map evolves from water-like to hard-sphere-like upon varying between 4/7 and 6/7. Thus, we traverse the range of liquid behavior encompassed by hard spheres $(\lambda=1)$ and water-like $(\lambda \sim 4 / 7)$ by simply varying the ratio of hard to soft-core diameters.

Further work is needed to establish whether a ratio of competing length scales close to 0.6 is generally associated with water-like anomalies in other core-softened potentials, e.g., achieving two characteristic length scales by using a linear combination of Gaussian [163] potentials of different widths.

In sum, motivated by the need to better understand the phenomenon of liquid polyamorphism [164, 165, 166], we are carrying out a systematic study of the effects of $\lambda$ and the ratio of characteristic energies on the existence of a LL transition, the positive or negative slope of the line of first-order LL transitions in the $(P, T)$ plane, and the relationship, if any [143, 144], between the LL transition and density anomalies. We will perform calculations in parallel for both confined and bulk water. 


\section{Understanding "Dynamic Heterogeneities"}

\section{Recent Experiments on Confined Water}

Simulations and experiments both are consistent with the possibility that the LL critical point, if it exists at all, lies in the experimentally inaccessible No-Man's land. If this statement is valid, then at least two reactions are possible:

(i) If something is not experimentally accessible, then it does not deserve discussion.

(ii) If something is not experimentally accessible, but its influence $i s$ experimentally accessible, then discussion is warranted

Option (ii) has guided most research thus far, since the manifestations of a critical point extend far away from the actual coordinates of that point. Indeed, accepting option (i) means there is nothing more to discuss. However if we confine water, the homogeneous nucleation temperature decreases and it becomes possible to enter the NoMan's land and, hence, search for the LL critical point. In fact, recent experiments at MIT and Messina by the Chen and Mallamace groups demonstrate that for nanopores of typically $1.5 \mathrm{~nm}$ diameter, the No-Man's land actually ceases to exist - one can supercool the liquid state all the way down to the glass temperature. Hence studying confined water offers the opportunity of directly testing, for the first time, the LL phase transition hypothesis.

In fact, using two independent techniques, neutron scattering and NMR, the MIT and Messina groups found a sharp kink in the dynamic properties (a "dynamic crossover") at the same temperature $T_{L} \approx 225 \mathrm{~K}[90$, 167]. Our calculations on bulk models [168] are not inconsistent with one tentative interpretation of this dynamic crossover as resulting from the system passing from the high-temperature high-pressure "HDL" side of the Widom line (where the liquid might display fragile behavior) to the low-temperature low-pressure "LDL" side of the Widom line (where the liquid might display strong behavior). By definition, the Widom line-defined to be the line in the pressure-temperature plane where the correlation length has its maximum - arises only if there is a critical point. Hence interpreting the MIT-Messina experiments in terms of a Widom line is of potential relevance to testing experimentally, for confined water, the liquid-liquid critical point hypothesis.

The interpretation of the dynamic crossover could have implications for nanofluidics and perhaps even for natural confined water systems, e.g., some proteins appear to undergo a change in their flexibility at approximately the same temperature $T_{L}$ that the MIT-Messina experiments identify for the dynamic crossover in pure confined water.

\section{Possible Significance of the Widom Line}

The conjectured interpretation of the MIT-Messina experiments relies on the concept of the Widom line, a concept not widely appreciated even though it has been known by experimentalists dating back to the $1958 \mathrm{Ph} . \mathrm{D}$. thesis of J. M. H. Levelt (now Levelt-Sengers) [171]. Since a Widom line arises only from a critical point, if the MIT-Messina experiments can be rationalized by a Widom line then they are consistent with the existence of a LL critical point in confined water.

By definition, in a first order phase transition, thermodynamic functions discontinuously change as we cool the system along a path crossing the equilibrium coexistence line [Fig. 2(a), path $\beta$ ]. However in a real experiment, this discontinuous change may not occur at the coexistence line since a substance can remain in a supercooled metastable phase until a limit of stability (a spinodal) is reached [4] [Fig. 2(b), path $\beta$ ].

If the system is cooled isobarically along a path above the critical pressure $P_{c}$ [Fig. 2(b), path $\alpha$ ], the state functions continuously change from the values characteristic of a high temperature phase (gas) to those characteristic of a low temperature phase (liquid). The thermodynamic response functions which are the derivatives of the state functions with respect to temperature [e.g., $C_{P}$ ] have maxima at temperatures denoted $T_{\max }(P)$. Remarkably these maxima are still prominent far above the critical pressure $[171,172]$, and the values of the response functions at $T_{\max }(P)$ (e.g., $C_{P}^{\max }$ ) diverge as the critical point is approached. The lines of the maxima for different response functions asymptotically approach one another as the critical point is approached, since all response functions become expressible in terms of the correlation length. This asymptotic line is sometimes called the Widom line, and is often regarded as an extension of the coexistence line into the "one-phase regime."

Suppose now that the system is cooled at constant pressure $P_{0}$. (i) If $P_{0}>P_{C}$ ("path $\alpha$ "), experimentallymeasured quantities will change dramatically but continuously in the vicinity of the Widom line (with huge fluctuations as measured by, e.g., $C_{P}$ ). (ii) If $P_{0}<P_{C}$ ("path $\beta$ "), experimentally-measured quantities will change discontinuously if the coexistence line is actually seen. However the coexistence line can be difficult to detect in a pure system due to metastability, and changes will occur only when the spinodal is approached where the gas phase is no longer stable.

In the case of water - the most important solvent for biological function $[173,174]$ - a significant change in dynamical properties has been suggested to take place in deeply supercooled states $[175,176,177,178]$. Unlike other network forming materials [179], water behaves as a fragile liquid in the experimentally accessible window $[176,180,181]$. Based on analogies with other 


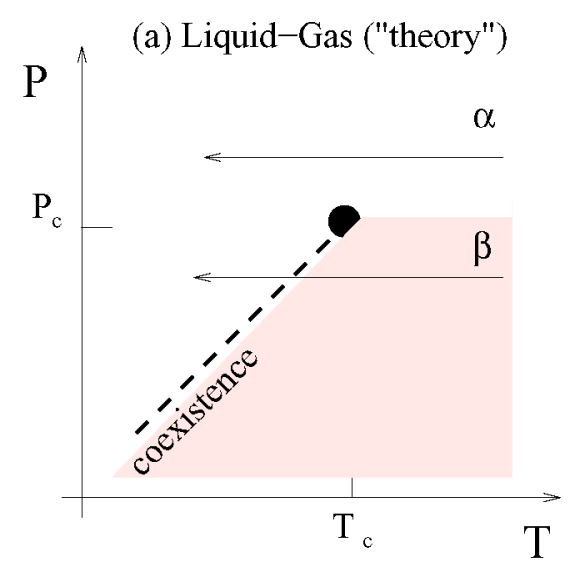

(c) Water

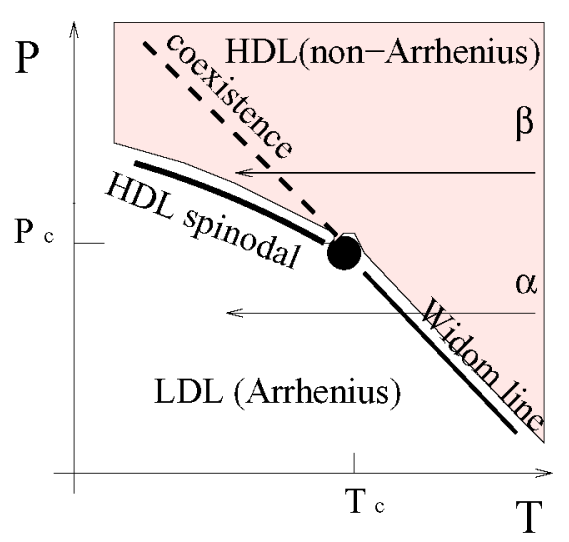

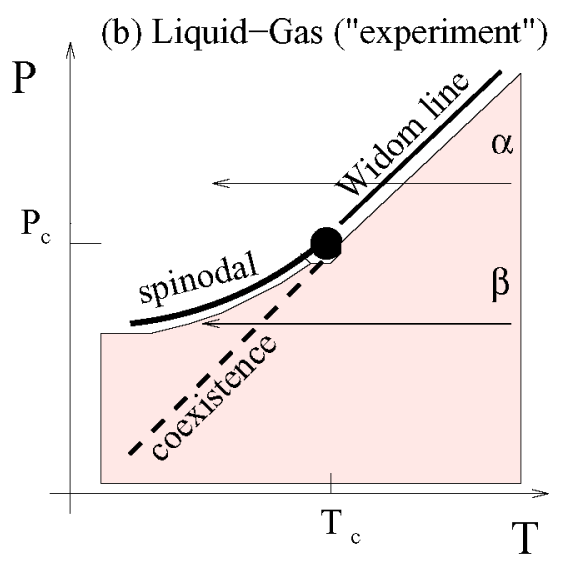

(d) Jagla Model

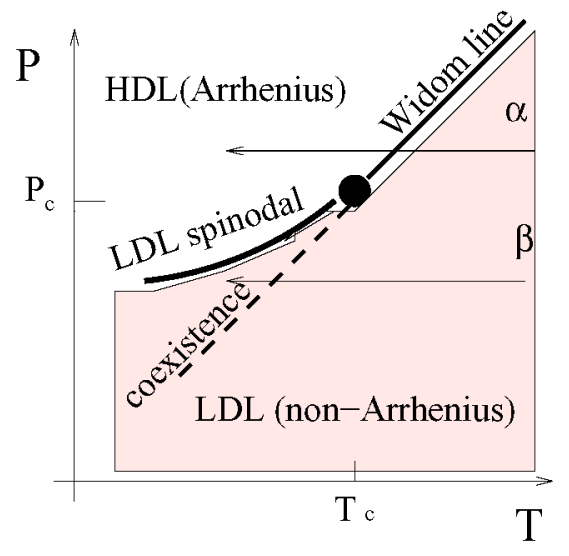

FIGURE 2. (a) Schematic phase diagram for the critical region associated with a liquid-gas critical point. Two features display mathematical singularities: the critical point and the liquid-gas coexistence. (b) Same, with the addition of the gas-liquid spinodal and the Widom line. Along the Widom line, thermodynamic response functions have extrema in their $T$ dependence. (c) A hypothetical phase diagram for water of possible relevance to recent confined water neutron scattering experiments $[169,167,170,90]$. (d) A sketch of the $P-T$ phase diagram for the two-scale Jagla model. 
network forming liquids and with the thermodynamic properties of the amorphous forms of water, it has been suggested that, at ambient pressure, liquid water should show a crossover between fragile behavior at high $\mathrm{T}$ to strong behavior at low $\mathrm{T}[182,177,138,139,183]$ in the deep supercooled region of the phase diagram below the homogeneous nucleation line. This region may contain the hypothesized LL critical point [44], the terminal point of a line of first order LL phase transitions. Recently, dynamic crossovers in confined water were studied experimentally [96, 184, 169, 90] since nucleation can be avoided in confined geometries. Also, a dynamic crossover has been associated with the LL phase transition in both silicon and silica $[185,186]$. In the following, we offer a very tentative interpretation of the observed fragility transition in water as arising from crossing the Widom line emanating from the hypothesized LL critical point [186] [Fig. 2, path $\alpha$ ].

\section{Methods Employed to Study Dynamic Crossovers in Confined Water}

Using MD simulations [187], we study three models, each of which has a LL critical point. Two of the models, (the TIP5P [68] and the ST2 [188]) treat water as a multiple site rigid body, interacting via electrostatic site-site interactions complemented by a Lennard-Jones potential. The third model is the spherically symmetric "two-scale" Jagla potential with attractive and repulsive ramps which has been recently studied in the context of LL phase transitions and liquid anomalies $[157,151]$. For all three models, we evaluate the loci of maxima of the relevant response functions, $K_{T}$ and $C_{P}$, which coincide close to the critical point and give rise to the Widom line. We carefully explore the hypothesis that, for all three potentials, a dynamic crossover occurs when the Widom line is crossed.

For TIP5P we find a LL critical point $[69,70]$, from which the Widom line develops [Fig.4(a)]. The coexistence curve is negatively sloped, so the Clapeyron equation implies that the high-temperature phase is a highdensity liquid (HDL) and the low-temperature phase is a low-density liquid (LDL). The diffusion coefficient $D$ is evaluated from the long time limit of the mean square displacement along isobars [Fig.4(b)]. We find that isobars crossing the Widom line (path $\alpha$ ) show a clear crossover (i) from a non-Arrhenius behavior at high $T$ [which can be well fitted by a power law function $\left.D \sim\left(T-T_{\mathrm{MCT}}\right)^{\gamma}\right]$, consistent with the mode coupling theory predictions [189]), (ii) to an Arrhenius behavior at low $T$ [which can be described by $\left.D \sim \exp \left(-E_{a} / T\right)\right]$. The crossover between these two functional forms takes place when crossing the Widom line.
For paths $\beta$, crystallization occurs in TIP5P [69], so the hypothesis that there is no fragility transition cannot be checked at low temperature. Hence we consider a related potential, ST2, for which crystallization is absent within the time scale of the simulation. Simulation details are described in [190]. This potential also displays a LL critical point [44, 190] [Fig.4(c)]. Along paths $\alpha$ a fragility transition may take place, while along paths $\beta$ the $T$ dependence of $D$ does not show any sign of crossover to Arrhenius behavior and the fragile behavior is retained down to the lowest studied temperature. Indeed, for paths $\beta$, the entire $T$ dependence can be fit by a power law $\left(T-T_{\mathrm{MCT}}\right)^{\gamma}[$ Fig.4(d)].

If indeed TIP5P and ST2 water models support the connection between the Widom line and the dynamic fragility transition, it is natural to ask which features of the water molecular potential are responsible for the properties of water, especially because water's unusual properties are shared by several other liquids whose inter-molecular potential has two energy (length) scales such as silicon and silica $[185,191,186]$. Hence we also investigate the two-scale spherically symmetric Jagla potential $[157,138,139]$, displaying-without the need to supercool-a LL coexistence line which, unlike water, has a positive slope, implying that the Widom line is now crossed along $\alpha$ paths with $P>P_{C}$ [Fig.4(e)]. We verify a crossover in the behavior of $D(T)$ when the Widom line $\left(C_{P}^{\max }\right.$ line $)$ is crossed, such that at high temperature, $D$ exhibits an Arrhenius behavior, while at low temperature it followa a non-Arrhenius behavior, consistent with a power law [Fig.4(f)]. Along a $\beta$ path $\left(P<P_{C}\right), D(T)$ appears to follow the Arrhenius behavior over the entire studied temperature range. Thus we test that the dynamic crossover coincides with the location of the $C_{P}^{\max }$ line, extending the conclusion of the TIP5P and ST2 potentials to a general two-scale spherically symmetric potential.

\section{Hamiltonian Model of Water}

In Ref. [148], we investigated the generality of the dynamic crossover in a Hamiltonian model of water which displays a liquid-liquid phase transition at low temperatures. We consider a cell model that reproduces the fluid phase diagram of water and other tetrahedral network forming liquids $[50,51,52,53]$. For sake of clarity, we focus on water to explain the motivation of the model. The model is based on the experimental observations that on decreasing $P$ at constant $T$, or on decreasing $T$ at constant $P$, (i) water displays an increasing local tetrahedrality [149], (ii) the volume per molecule increases at sufficiently low $P$ or $T$, and (iii) the O-O-O angular correlation increases [74], consistent with simulations [150].

The system is divided into cells $i \in[1, \ldots, N]$ on a regular square lattice, each containing a molecule with 
volume $v \equiv V / N$, where $V \geq N v_{0}$ is the total volume of the system, and $v_{0}$ is the hard-core volume of one molecule. The cell volume $v$ is a continuous variable that gives the mean distance $r \equiv v^{1 / d}$ between molecules in $d$ dimensions. The van der Waals attraction between the molecules is represented by a truncated Lennard-Jones potential with characteristic energy $\varepsilon>0$

$$
U(r) \equiv \begin{cases}\infty & \text { for } \quad r \leq R_{0} \\ \varepsilon\left[\left(\frac{R_{0}}{r}\right)^{12}-\left(\frac{R_{0}}{r}\right)^{6}\right] & \text { for } \quad r>R_{0},\end{cases}
$$

where $R_{0} \equiv v_{0}^{1 / d}$ is the hard-core distance [148].

Each molecule $i$ has four bond indices $\sigma_{i j} \in[1, \ldots, q]$, corresponding to the nearest-neighbor cells $j$. When two nearest-neighbor molecules have the facing $\sigma_{i j}$ and $\sigma_{j i}$ in the same relative orientation, they decrease the energy by a constant $J$, with $0<J<\varepsilon$, and form a bond, e.g. a (non-bifurcated) hydrogen bond for water, or a ionic bond for $\mathrm{SiO}_{2}$. The choice $J<\varepsilon$ guarantees that bonds are formed only in the liquid phase. Bonding and intramolecular (IM) interactions are accounted for by the two Hamiltonian terms

$$
\mathscr{H}_{\mathrm{B}} \equiv-J \sum_{\langle i, j\rangle} \delta_{\sigma_{i j} \sigma_{j i}}
$$

where the sum is over n.n. cells, $0<J<\varepsilon$ is the bond energy, $\delta_{a, b}=1$ if $a=b$ and $\delta_{a, b}=0$ otherwise, and

$$
\mathscr{H}_{\mathrm{IM}} \equiv-J_{\sigma} \sum_{i} \sum_{(k, \ell)_{i}} \delta_{\sigma_{i k} \sigma_{i \ell}}
$$

where $\sum_{(k, \ell)_{i}}$ denotes the sum over the IM bond indices $(k, l)$ of the molecule $i$ and $J_{\sigma}>0$ is the IM interaction energy with $J_{\sigma}<J$, which models the angular correlation between the bonds on the same molecule. The total energy of the system is the sum of the van der Waals interaction and Eqs. (2) and (3).

We find that different response functions such as $C_{P}$, $\alpha_{p}$ (see Fig. 3) show maxima and these maxima increase and seem to diverge as the critical pressure is approached, consistent with the picture of Widom line that we discussed for other water models in the sections above. Moreover we find that the temperature derivative of the number of hydrogen bonds $d N_{H B} / d T$ displays a maximum in the same region where the other thermodynamic response functions have maxima; suggesting that the fluctuations in the number of hydrogen bonds is maximum at the Widom line temperature $T_{W}$.

To futher test if this model system also displays a dynamic crossover as found in the other models of water, we study the total spin relaxation time of the system as a function of $T$ for different pressures. We find that for $J_{\sigma} / \varepsilon=0.05$ (liquid-liquid critical point scenario) the crossover occurs at $T_{W}(P)$ for $P<P_{C^{\prime}}$ [Fig. 5(a)]. For completeness we study the system also in the case of singularity free scenario, corresponding to $J_{\sigma}=0$. For $J_{\sigma}=0$ the crossover is at $T\left(C_{P}^{\max }\right)$, the temperature of $C_{P}^{\max }[$ [Fig. 5(b)].

We next calculate the Arrhenius activation energy $E_{\mathrm{A}}(P)$ from the low- $T$ slope of $\log \tau$ vs. $1 / T$ [Fig. 6(a)]. We extrapolate the temperature $T_{\mathrm{A}}(P)$ at which $\tau$ reaches a fixed macroscopic time $\tau_{\mathrm{A}} \geq \tau_{\mathrm{C}}$. We choose $\tau_{\mathrm{A}}=10^{14}$ MC steps $>100 \mathrm{sec}$ [131] [Fig. 6(b)]. We find that $E_{\mathrm{A}}(P)$ and $T_{\mathrm{A}}(P)$ decrease upon increasing $P$ in both scenarios, providing no distinction between the two interpretations. Instead, we find a dramatic difference in the $P$ dependence of the quantity $E_{\mathrm{A}} /\left(k_{B} T_{\mathrm{A}}\right)$ in the two scenarios, increasing for the LL critical point scenario and approximately constant for the singularity free scenario [Fig. 6(c)].

\section{Glass Transition in Biomolecules}

Next we explore the hypothesis [211] that the observed glass transition in biomolecules [198, 212, 213, 214, $215,216,217,218,219,220,221,222,223,224]$ is related to the liquid-liquid phase transition using molecular dynamics (MD) simulations. Specifically, Kumar et al. [211] studied the dynamic and thermodynamic behavior of lysozyme and DNA in hydration TIP5P water, by means of the software package GROMACS [225] for (i) an orthorhombic form of hen egg-white lysozyme [226] and (ii) a Dickerson dodecamer DNA [227] at constant pressure $P=1 \mathrm{~atm}$, several constant temperatures $T$, and constant number of water molecules $N$ (NPT ensemble).

The simulation results for the mean square fluctuations $\left\langle x^{2}\right\rangle$ of both protein and DNA are shown in Figure 7(a). Kumar et al. calculated the mean square fluctuations $\left\langle x^{2}\right\rangle$ of the biomolecules from the equilibrated configurations, first for each atom over $1 \mathrm{~ns}$, and then averaged over the total number of atoms in the biomolecule. They find that $\left\langle x^{2}\right\rangle$ changes its functional form below $T_{\mathrm{p}} \approx 245 \mathrm{~K}$, for both lysozyme [Fig. 7(a)] and DNA [Fig. 7(b)].

Kumar et al. next calculated $C_{P}$ by numerical differentiation of the total enthalpy of the system (protein and water) by fitting the simulation data for enthalpy with a fifth order polynomial, and then taking the derivative with respect to $T$. Figures 8 (a) and 8 (b) display maxima of $C_{P}(T)$ at $T_{\mathrm{W}} \approx 250 \pm 10 \mathrm{~K}$ for both biomolecules.

Further, to describe the quantitative changes in structure of hydration water, Kumar et al. calculated the local tetrahedral order parameter $Q[136,160,161,162]$ for hydration water surrounding lysozyme and DNA. Figures $8(\mathrm{c})$ and 8 (d) show that the rate of increase of $Q$ has a maximum at $245 \pm 10 \mathrm{~K}$ for lysozyme and DNA hydration water respectively; the same temperatures of the crossover in the behavior of mean square fluctuations. 

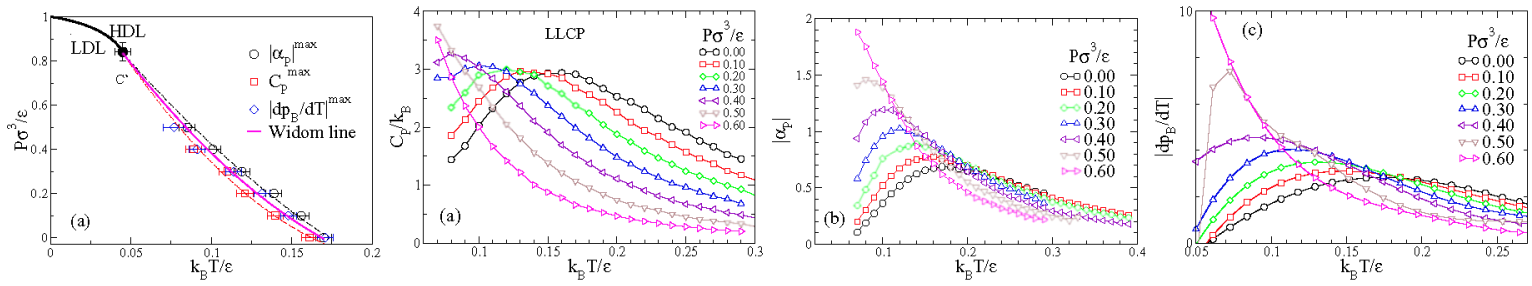

FIGURE 3. (a) Phase diagram below $T_{\mathrm{MD}}$ line shows that $\mid d p_{\mathrm{B}} / d T^{\max }(\diamond)$ coincides with the Widom line $T_{W}(P)$ (solid line) within error bars: $C^{\prime}$ is the HDL-LDL critical point, end of first-order phase transition line (thick line) [148]; symbols are maxima for $N=3600$ of $\left|\alpha_{P}\right|^{\max }(\bigcirc), C_{P}^{\max }(\square)$, and $\left|d p_{\mathrm{B}} / d T\right|^{\max }(\diamond)$; upper and lower dashed line are quadratic fits of $\left|\alpha_{P}\right|^{\max }$ and $C_{P}^{\max }$, respectively, consistent with $C^{\prime} ;\left|\alpha_{P}\right|^{\max }$ and $C_{P}^{\max }$ are consistent within error bars. Maxima are estimated from panels (b), (c) and (d), where each quantity is shown as functions of $T$ for different $P<P_{C^{\prime}}$. In (e) $\left|d p_{\mathrm{B}} / d T\right|^{\text {max }}$ is the numerical derivative of $p_{\mathrm{B}}$ from simulations in (d).

Upon cooling, the diffusivity of hydration water exhibits a dynamic crossover from non-Arrhenius to Arrhenius behavior at the crossover temperature $T_{\times} \approx 245 \pm$ $10 \mathrm{~K}$ [Figure $8(\mathrm{e})]$. The coincidence of $T_{\times}$with $T_{\mathrm{p}}$ within the error bars indicates that the behavior of the protein is strongly coupled with the behavior of the surrounding solvent, in agreement with recent experiments [198]. Note that $T_{\times}$is much higher than the glass transition temperature, estimated for TIP5P as $T_{g}=215 \mathrm{~K}$. Thus this crossover is not likely to be related to the glass transition in water.

The fact that $T_{\mathrm{p}} \approx T_{\times} \approx T_{\mathrm{W}}$ is evidence of the correlation between the changes in protein fluctuations [Figure $7(a)$ and the hydration water thermodynamics [Figure 8(a)]. Thus these results are consistent with the possibility that the protein glass transition is related to the Widom line (and hence to the hypothesized liquid-liquid critical point). Crossing the Widom line corresponds to a continuous but rapid transition of the properties of water from those resembling the properties of a local HDL structure for $T>T_{\mathrm{W}}(P)$ to those resembling the properties of a local LDL structure for $T<T_{\mathrm{W}}(P)$. A consequence is the expectation that the fluctuations of the protein residues in predominantly LDL-like water (more ordered and more rigid) just below the Widom line should be smaller than the fluctuations in predominantly HDLlike water (less ordered and less rigid) just above the Widom line.

The quantitative agreement of the results for both DNA and lysozyme [Figures 3 and 4] suggests that it is indeed the changes in the properties of hydration water that are responsible for the changes in dynamics of the protein and DNA biomolecules. Our results are in qualitative agreement with recent experiments on hydrated protein and DNA [228] which found the crossover in side-chain fluctuations at $T_{\mathrm{p}} \approx 225 \mathrm{~K}$.

\section{Outlook}

It is possible that other phenomena that appear to occur on crossing the Widom line are in fact not coincidences, but are related to the changes in local structure that occur when the system changes from the "HDL-like" side to the "LDL-like" side. In this work we concentrated on reviewing the evidence for changes in dynamic transport properties, such as diffusion constant and relaxation time. Additional examples include: (1) a breakdown of the Stokes-Einstein relation for $T<T_{W}(P)[192,193$ ? , 195, 196, 197], (2) systematic changes in the static structure factor $S(q)$ and the corresponding pair correlation function $g(r)$ revealing that for $T<T_{W}(P)$ the system more resembles the structure of LDL than HDL, (3) appearance for $T<T_{W}(P)$ of a shoulder in the dynamic structure factor $S(q, \omega)$ at a frequency $\omega \approx 60 \mathrm{~cm}^{-1} \approx 2$ THz [198, 199], (4) rapid increase in hydrogen bonding degree for $T<T_{W}(P)$ [200, 201], (5) a minimum in the density at low temperature [202], and (6) a scaled equation of state near the critical point [203]. It is important to know how general a given phenomenon is, such as crossing the Widom line which by definition is present whenever there is a critical point. Using data on other liquids which have local tetrahedral symmetry, such as silicon and silica, which appear to also display a liquidliquid critical point and hence must possess a Widom line emanating from this point into the one-phase region. For example, we learned of interesting new work on silicon, which also interprets structural changes as arising from crossing the Widom line of silicon [204]. It might be interesting to test the effect of the Widom line on simple model systems that display a liquid-liquid critical point, such as two-scale symmetric potentials of the sort recently studied by Xu and her collaborators [205] or by Franzese [147] and Barros de Oliveira and coworkers [156].

Very recently, Mallamace and his collaborators succeeded in locating the Widom line by finding a clearcut maximum in the coefficient of thermal expansion, at 

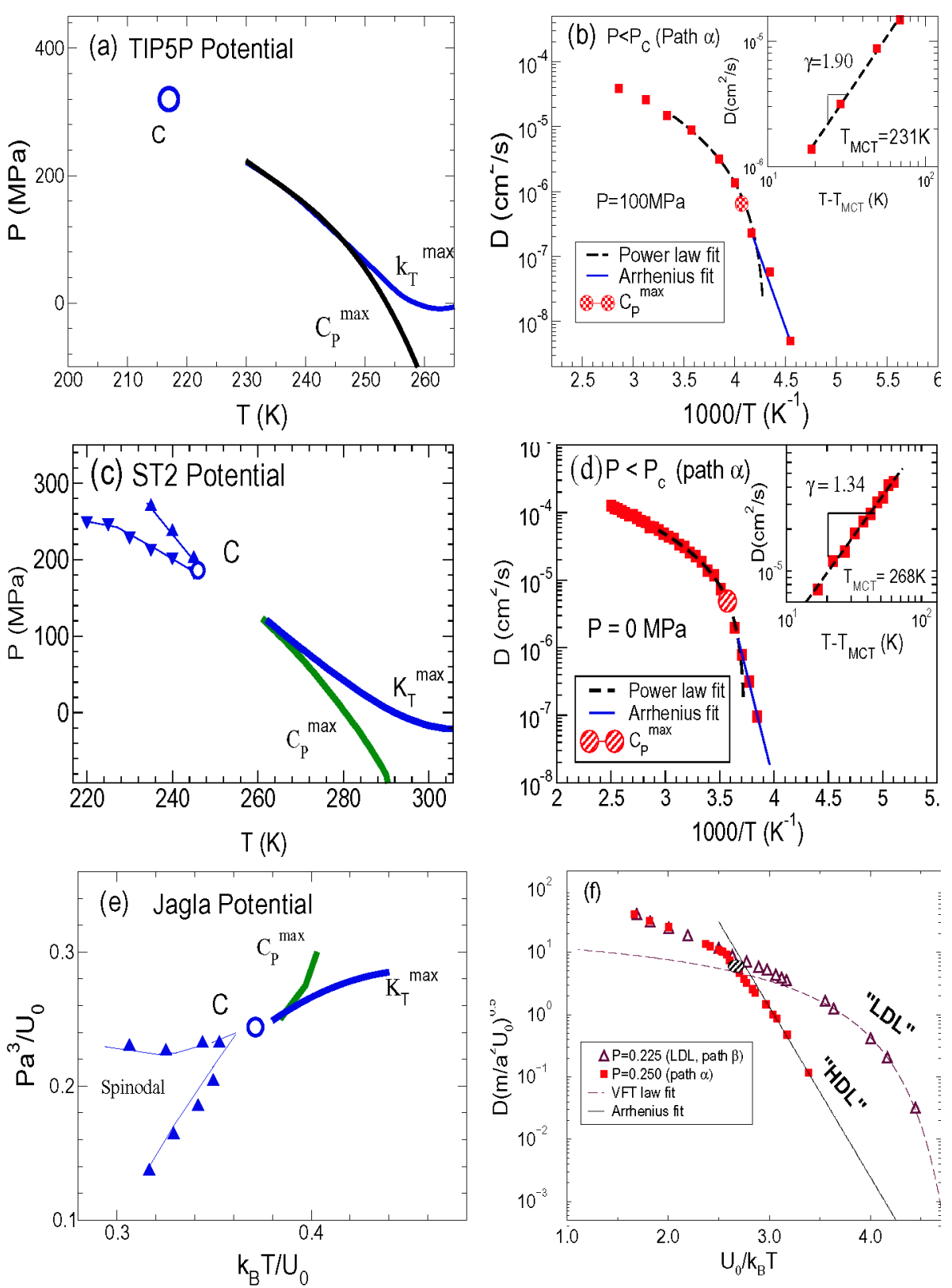

FIGURE 4. (a) Relevant part of the phase diagram for the TIP5P potential, showing the liquid-liquid critical point $C$ at $P_{C}=320 \mathrm{MPa}$ and $T_{C}=217 \mathrm{~K}$, the line of maximum of isobaric specific heat $C_{P}^{\max }$ and the line of maximum of isothermal compressibility $K_{T}^{\max }$. (b) $D$ as a function of $T$ for $P=100 \mathrm{MPa}$ (path $\alpha$ ). At high temperatures, $D$ behaves like that of a nonArrhenius liquid and can be fit by $D \sim\left(T-T_{M C T}\right)^{\gamma}$ (also shown in the inset) where $T_{M C T}=220 \mathrm{~K}$ and $\gamma=1.942$, while at low temperatures the dynamic behavior changes to that of a liquid where $D$ is Arrhenius. (c) The same for the ST2 potential. The liquid-liquid critical point $C$ is located at $P_{C}=246 \mathrm{MPa}$ and $T_{C}=146 \mathrm{~K}$. (d) $D$ as a function of $T$ for $P=100 \mathrm{MPa}$ (path $\alpha$ ). At high temperatures, $D$ behaves like that of a non-Arrhenius liquid and can be fit by $D \sim\left(T-T_{M C T}\right)^{\gamma}$ (also shown in the inset) where $T_{M C T}=239 \mathrm{~K}$ and $\gamma=1.57$, while at low temperatures the dynamic behavior changes to that of a liquid where $D$ is Arrhenius. (e) Phase diagram for the Jagla potential in the vicinity of the liquid-liquid phase transition. Shown are the liquid-liquid critical point $C$ located at $P_{c}=0.24$ and $T_{C}=0.37$, the line of isobaric specific heat maximum $C_{P}^{\max }$, the line of isothermal compressibility $K_{T}^{\max }$, and spinodal lines. (f) $D$ as a function of $T$ for $P=0.250$ (squares, path $\alpha$ ) and $P=0.225$ (triangles, path $\beta$ ). Along path $\alpha$, one can see a sharp crossover from the high temperature Arrhenius behavior $D \approx \exp (-1.53 / T)$ with lower activation energy to a low temperature Arrhenius behavior $D \approx \exp (-6.3 / T)$ with high activation energy, which is a characteristic of the HDL. Along path $\beta$, there is no sharp changes near the critical point, because the liquid remains in the LDL phase. However, near the glass transition, LDL exhibits a non-Arrhenius behavior characterized by the VFT fit at very low temperature. 

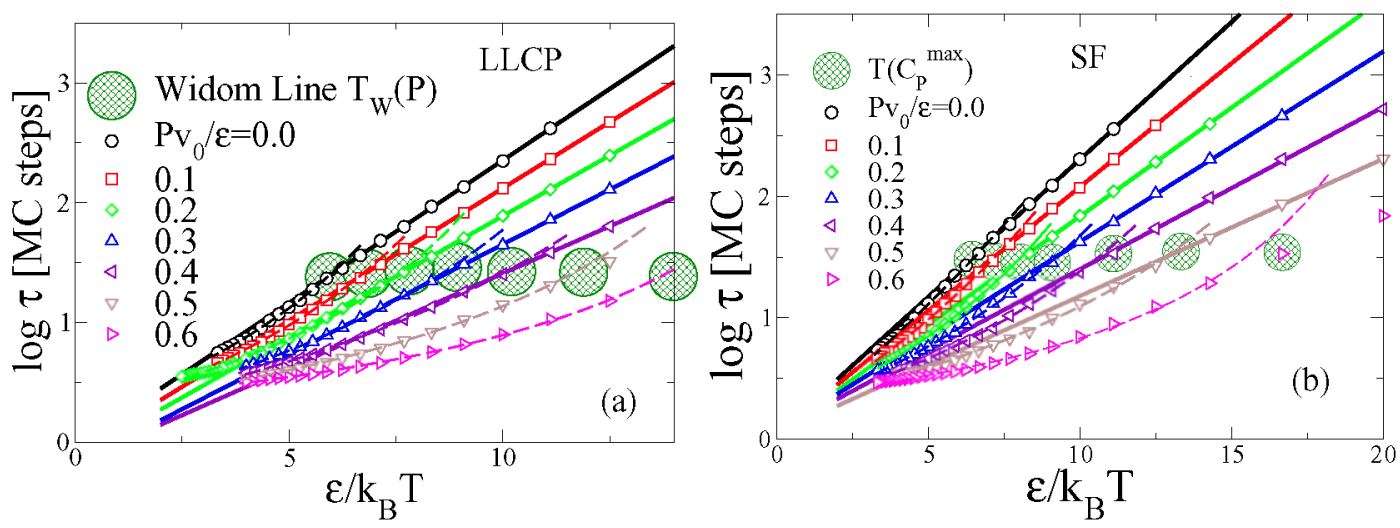

FIGURE 5. Dynamic crossover - large hatched circles of a radius approximately equal to the error bar-in the orientational relaxation time $\tau$ for a range of different pressures. (a) The LL critical point (LLCP) scenario, with crossover temperature at $T_{W}(P)$. (b) The singularity free (SF) scenario, with crossover temperature at $T\left(C_{P}^{\max }\right)$. Solid and dashed lines represent Arrhenius and non-Arrhenius fits, respectively. Notice that the dynamic crossover occurs at approximately the same value of $\tau$ for all seven values of pressure studied.
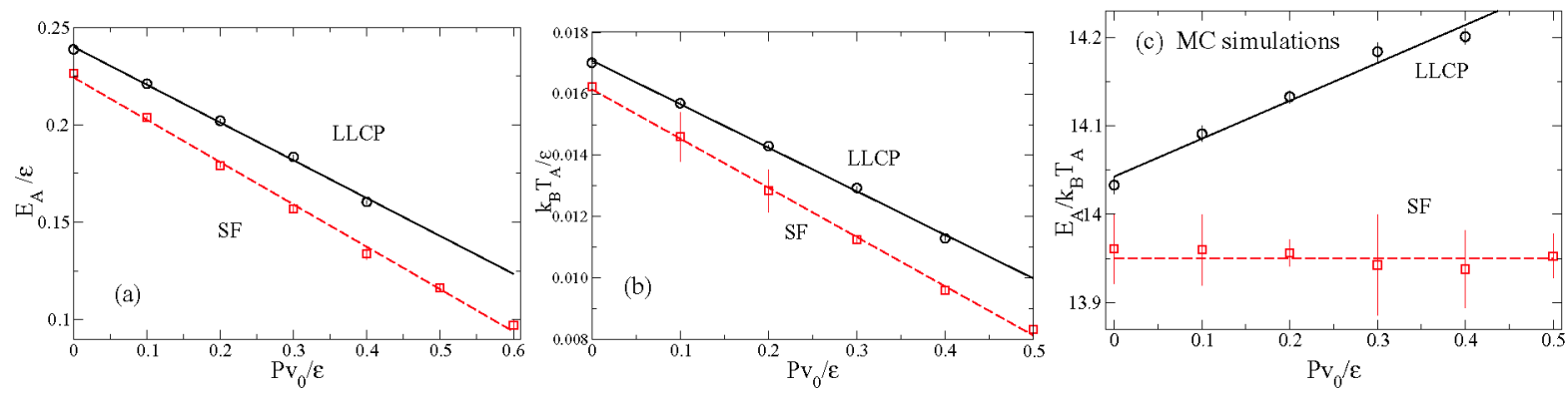

FIGURE 6. Effect of pressure on the activation energy $E_{\mathrm{A}}$. (a) Demonstration that $E_{\mathrm{A}}$ decreases linearly for increasing $P$ for both the LL critical point and the singularity free scenarios. The lines are linear fits to the simulation results (symbols). (b) $T_{\mathrm{A}}$, defined such that $\tau\left(T_{\mathrm{A}}\right)=10^{14} \mathrm{MC}$ steps $>100 \mathrm{sec}$ [131], decreases linearly with $P$ for both scenarios. (c) $P$ dependence of the quantity $E_{\mathrm{A}} /\left(k_{B} T_{\mathrm{A}}\right)$ is different in the two scenarios. In the LL critical point (LLCP) scenario, $E_{\mathrm{A}} /\left(k_{B} T_{\mathrm{A}}\right)$ increases with increasing $P$, and it is approximately constant in the singularity free (SF) scenario. The lines are guides to the eyes. 

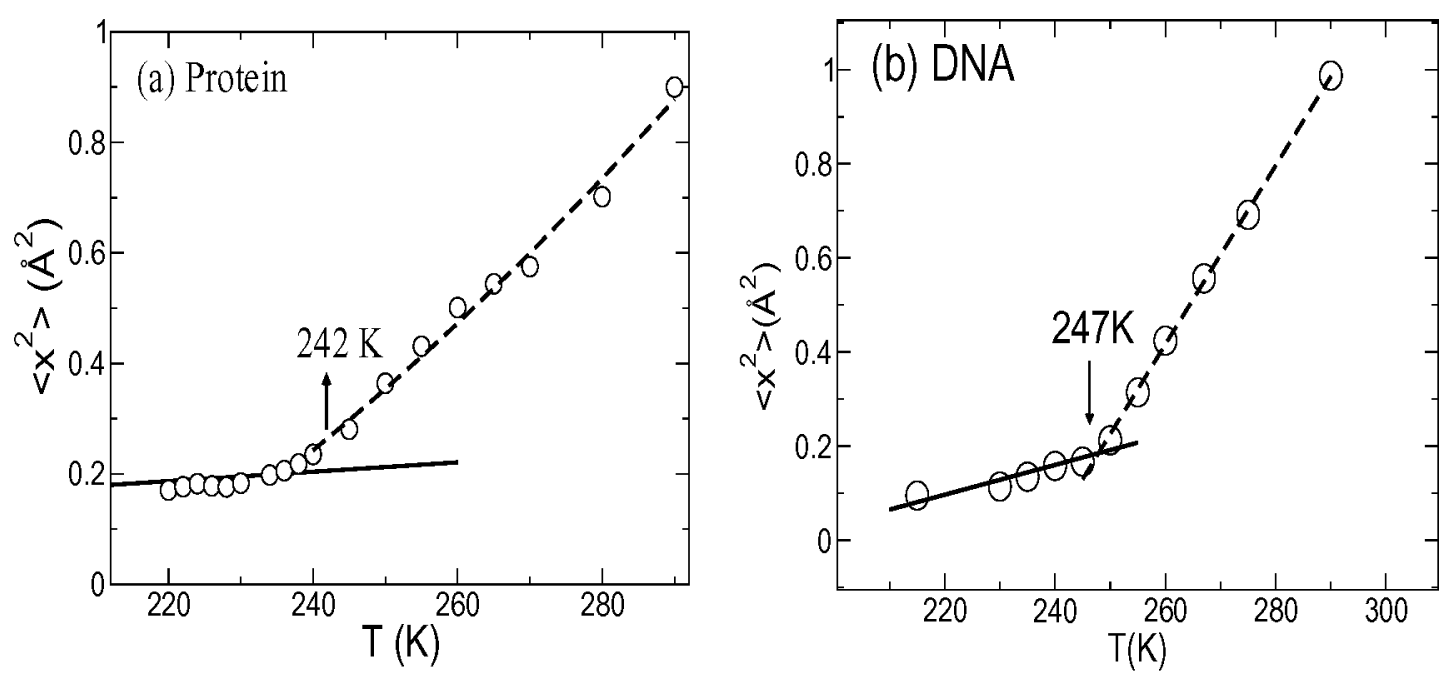

FIGURE 7. Mean square fluctuation of (a) lysozyme, and (b) DNA showing that there is a transition around $T_{\mathrm{p}} \approx 242 \pm 10 \mathrm{~K}$ for lysozyme and around $T_{\mathrm{p}} \approx 247 \pm 10 \mathrm{~K}$ for DNA. For very low $T$ one would expect a linear increase of $\left\langle x^{2}\right\rangle$ with $T$, as a consequence of harmonic approximation for the motion of residues. At high $\mathrm{T}$, the motion becomes non-harmonic and we fit the data by a polynomial. We determine the dynamic crossover temperature $T_{\mathrm{p}}$ from the crossing of the linear fit for low $\mathrm{T}$ and the polynomial fit for high $\mathrm{T}$. We determine the error bars by changing the number of data points in the two fitting ranges.

$T_{W} \approx 225 \mathrm{~K}[206,207,208]$, which remarkably is the same temperature as the specific heat maximum [209]. Also, private discussions with Jacob Klein reveal a possible reason for why confined water does not freeze at $-38 \mathrm{C}$, the bulk homogeneous nucleation temperature: Klein and co-workers [210] noted that confined water behaves differently than typical liquids in that it water does not experience the huge increase in viscosity characteristic of other strongly confined liquids. They interpret this experimental finding as arising from the fact that strong confinement hampers the formation of a hydrogen bonded network, and we know from classic work of Linus Pauling that without the extensive hydrogen bonded network, water's freezing temperature will be depressed by more than 100 degrees. Thus confinement reduces the extent of the hydrogen bonded network and hence lowers the freezing temperature, but leaves the key tetrahedral local geometry of the water molecule itself unchanged.

\section{Acknowledgments}

This work has been supported by the NSF and DOE.

\section{REFERENCES}

1. R. Waller, trans., Essayes of Natural Experiments [original in Italian by the Secretary of the Academie del Cimento]. Facsimile of 1684 English translation (Johnson Reprint Corporation, New York, 1964).
2. H. E. Stanley, "Unsolved Mysteries of Water in its Liquid and Glass Phases" [edited transeript of Turnbull Prize lecture], Materials Research Bulletin 24, No. 5, 22-30 (May 1999).

3. C. A. Angell, M. Oguni, and W. J. Sichina, "Heat Capacity of Water at Extremes of Supercooling and Superheating," J. Phys. Chem. 86, $998-1002$ (1982).

4. P. G. Debenedetti and H. E. Stanley, "The Physics of Supercooled and Glassy Water," Physics Today 56[issue 6], 40-46 (2003).

5. O. Mishima and H. E. Stanley, "The relationship between liquid, supercooled and glassy water" [invited review article], Nature 396, 329-335 (1998).

6. R. J. Speedy and C. A. Angell, "Isothermal Compressibility of Supercooled Water and Evidence for a Thermodynamic Singularity," J. Chem. Phys. 65, 851-858 (1976)

7. E. F. Burton and W. F. Oliver, "The Crystal Structure of Ice at Low Temperatures," Proc. Roy. Soc. London Ser. A 153, 166-172 (1936).

8. O. Mishima, L. D. Calvert, and E. Whalley, "'Melting' Ice I at $77 \mathrm{~K}$ and $10 \mathrm{kbar}$ : A New Method of Making Amorphous Solids," Nature 310, 393-395 (1984).

9. H.-G. Heide, "Observations on Ice Layers," Ultramicroscopy 14, 271-278 (1984).

10. O. Mishima, L. D. Calvert, and E. Whalley, "An Apparently First-Order Transition between Two Amorphous Phases of Ice Induced by Pressure," Nature 314, 76-78 (1985).

11. M. C. Bellissent-Funel, L. Bosio, A. Hallbrucker, E. Mayer, and R. Sridi-Dorbez, "X-Ray and Neutron Scattering Studies of the Structure of Hyperquenched Glassy Water," J. Chem. Phys. 97, 1282-1286 (1992).

12. M. C. Bellissent-Funel and L. Bosio, "A Neutron Scattering Study of Liquid $\mathrm{D}_{2} \mathrm{O}$," J. Chem. Phys. 102, 3727-3735 (1995). 

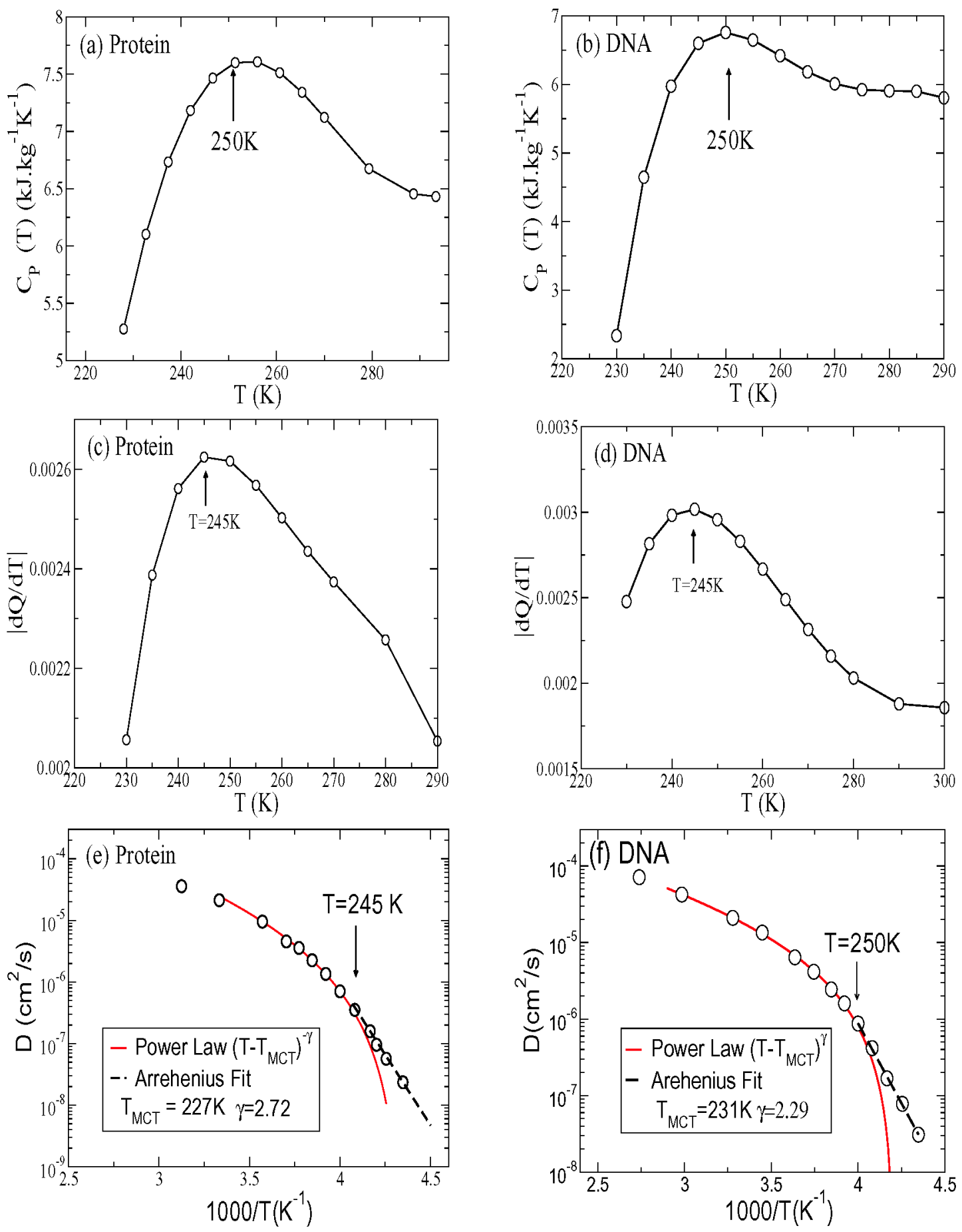

FIGURE 8. The specific heat of the combined system (a) lysozyme and water, and (b) DNA and water, display maxima at $250 \pm 10 \mathrm{~K}$ and $250 \pm 12 \mathrm{~K}$ respectively, which are coincident within the error bars with the temperature $T_{\mathrm{p}}$ where the crossover in the behavior of $\left\langle x^{2}\right\rangle$ is observed in Figure 7. Derivative with respec $t$ to temperature of the local tetrahedral order parameter $Q$ for (c) lysozyme and (d) DNA hydration water. A maximum in $|d Q / d T|$ at Widom line temperature suggests that the rate of change of local tetrahedrality of hydration water has a maximum at the Widom line. Diffusion constant of hydration water surrounding (e) lysozyme, and (f) DNA shows a dynamic transition from a power law behavior to an Arrhenius behavior at $T_{\times} \approx 245 \pm 10 \mathrm{~K}$ for lysozyme and $T_{\times} \approx 250 \pm 10 \mathrm{~K}$ for DNA, around the same temperatures where the behavior of $\left\langle x^{2}\right\rangle$ has a crossover, and $C_{P}$ and $|d Q / d T|$ have maxima. 
13. K. H. Smith, E. Shero, A. Chizmeshya, and G. H. Wolf, "The Equation of State of Polyamorphic Germania Glass: A Two-Domain Description of the Viscoelastic Response," J. Chem. Phys. 102, 6851-6857 (1995).

14. P. H. Poole, T. Grande, F. Sciortino, H. E. Stanley, and C. A. Angell, "Amorphous Polymorphism," J. Comp. Mat. Sci. 4, 373-382 (1995).

15. J. L. Finney, "Water? What's So Special About It? Phil. Trans. R. Soc. Lond. B: Biol. Sci. 359, 1145-1163 (2004).

16. E. Mayer, "Hyperquenched Glassy Bulk Water: A Comparison with Other Amorphous Forms of Water, and with Vitreous but Freezable Water in a Hydrogel and on Hydrated Methemoglobin," in Hydrogen Bond Networks, edited by M.-C. Bellissent-Funel and J. C. Dore (Kluwer Academic Publishers, Dordrecht, 1994), pp. 355-372.

17. O. Mishima, "Relationship between Melting and Amorphization of Ice," Nature 384, 546-549 (1996).

18. G. P. Johari, A. Hallbrucker, and E. Mayer, "Two Calorimetrically Distinct States of Liquid Water below 150 Kelvin," Science 273, 90-92 (1996).

19. U. Essmann and A. Geiger, "Molecular-Dynamics Simulation of Vapor-Deposited Amorphous Ice," $J$. Chem. Phys. 103, 4678-4692 (1995).

20. T. Loerting, C. Salzmann, I. Kohl, E. Mayer, and A. Hallbrucker, "A Second Structural State of High-Density Amorphous Ice at $77 \mathrm{~K}$ and 1 bar," Phys. Chem. Chem. Phys. 3, 5355 (2001).

21. J. L. Finney, D. T. Bowron, A. K. Soper, T. Loerting, E. Mayer, and A. Hallbrucker, "Structure of a New Dense Amorphous Ice," Phys. Rev. Lett. 89, 503-506 (2002).

22. J. S. Tse, D. D. Klug, M. Guthrie, C. A. Tulk, C. J. Benmore, and J. Urquidi, "Investigation of the Intermediate- and High-Density Forms of Amorphous Ice by Molecular Calculations Dynamics and Diffraction Experiments," Phys. Rev. B 71, 214107 (2005).

23. I. Brovchenko, A. Geiger, and A. Oleinikova, "Multiple Liquid-Liquid Transitions in Supercooled Water," J. Chem. Phys. 118, 9473-9476 (2003).

24. I. Brovchenko, A. Geiger, and A. Oleinikova, "LiquidLiquid Phase Transitions in Supercooled Water Studied by Computer Simulations of Various Water Models," J. Chem. Phys. 123, 044515 (2005).

25. P. Jedlovszky and R. Vallauri, "Liquid-Vapor and Liquid-Liquid Phase Equilibria of the BrodholtSampoli-Vallauri Polarizable Water Model," J. Chem. Phys. 122, 081101 (2005).

26. J. A. White, "Multiple Critical Points for Square-Well Potential with Repulsive Shoulder," Physica A 346, 347-357 (2004).

27. J. K. Christie, M. Guthrie, C. A. Tulk, C. J. Benmore, D. D. Klug, S. N. Taraskin, and S. R. Eliot, "Modeling the atomic structure of very high-density amorphous ice," Phys. Rev. B 72, 012201 (2005).

28. J. L. Finney, A. Hallbrucker, I. Kohl, A. K. Soper, and D. T. Bowron, "Structures of High and Low Density Amorphous Ice by Neutron Diffraction," Phys. Rev. Lett. 88, 225503 (2002).

29. D. Eisenberg and W. Kauzmann, The Structure and Properties of Water (Oxford University Press, New York, 1969).

30. J. D. Bernal and R. H. Fowler, "A Theory of Water and
Ionic Solution, with Particular Reference to Hydrogen and Hydroxyl Ions," J. Chem. Phys. 1, 515-548 (1933).

31. J. A. Pople, "Molecular Association in Liquids. II. A Theory of the Structure of Water," Proc. Roy. Soc. Lond. Ser. A 205, 163-178 (1951).

32. H. S. Frank and W.-Y. Wen, "Structural Aspects of IonSolvent Interaction in Aqueous Solutions: A Suggested Picture of Water Structure," Disc. Faraday Soc. 24, 133-140 (1957).

33. G. Némethy and H. A. Scheraga, "Structure of Water and Hydrophobic Bonding in Proteins: I. A Model for the Thermodynamic Properties of Liquid Water," $J$. Chem. Phys. 36, 3382-3400 (1962).

34. B. Kamb, "Ice Polymorphism and the Structure of Water," in Structural Chemistry and Molecular Biology, edited by A. Rich and N. Davidson (Freeman, San Francisco, 1968), pp. 507-542.

35. W. C. Röntgen, "Ueber die constitution des flüssigen wassers," Ann. d. Phys. u. Chem. 45, 91-97 (1892).

36. L. Pauling, "The Structure of Water," in Hydrogen Bonding, edited by D. Hadzi (Pergamon Press, New York, 1959), pp. 1-5.

37. R. J. Speedy, "Stability-Limit Conjecture: An Interpretation of the Properties of Water," J. Phys. Chem. 86, 982-991 (1982).

38. S. Sastry, P. Debenedetti, F. Sciortino, and H. E. Stanley, "Singularity-Free Interpretation of the Thermodynamics of Supercooled Water," Phys. Rev. E 53, 6144-6154 (1996). Citations: 125

39. H. E. Stanley, J. Teixeira, A.Geiger, and R.L.Blumberg, "Interpretation of the unusual behavior of $\mathrm{H}_{2} \mathrm{O}$ and $\mathrm{D}_{2} \mathrm{O}$ at low temperature: Are Concepts of Percolation Relevant to the 'Puzzle of Liquid Water'?" Invited Talk, INTERN'L CONFERENCE ON STATISTICAL MECHANICS, published in Physica A 106, 260-277 (1981).

40. H. E. Stanley, "A Polychromatic Correlated-Site Percolation Problem with Possible Relevance to the Unusual behavior of Supercooled $\mathrm{H}_{2} \mathrm{O}$ and $\mathrm{D}_{2} \mathrm{O}$," J. Phys. A 12, L329-L337 (1979).

41. H. E. Stanley and J. Teixeira, "Interpretation of The Unusual Behavior of $\mathrm{H}_{2} \mathrm{O}$ and $\mathrm{D}_{2} \mathrm{O}$ at Low Temperatures: Tests of a Percolation Model," J. Chem. Phys. 73, 3404-3422 (1980).

42. A. Geiger and H. E. Stanley, "Low-density patches in the hydrogen-bonded network of liquid water: Evidence from molecular dynamics computer simulations" Phys. Rev. Lett. 49, 1749-1752 (1982).

43. J. R. Errington, P. G. Debenedetti, and S. Torquato, "Cooperative Origin of Low-Density Domains in Liquid Water," Phys. Rev. Lett. 89, 215503 (2002).

44. P. H. Poole, F. Sciortino, U. Essmann, H. E. Stanley, "Phase Behavior of Metastable Water," Nature 360, 324-328 (1992).

45. E. G. Ponyatovskii, V. V. Sinitsyn and T. A. Pozdnyakova, "Second Critical Point and LowTemperature Anomalies in the Physical Properties of Water," JETP Lett. 60, 360-364 (1994).

46. C. T. Moynihan, "Two Species/Nonideal Solution Model for Amorphous/Amorphous Phase Transition." Mat. Res. Soc. Symp. Proc. 455, 411-425 (1997).

47. P. H. Poole, F. Sciortino, T.Grande, H. E. Stanley and C. A. Angell, "Effect of Hydrogen Bonds on the 
Thermodynamic Behavior of Liquid Water," Phys. Rev. Lett. 73, 1632-1635 (1994).

48. S. S. Borick, P. G. Debenedetti, and S. Sastry, "A Lattice Model of Network-Forming Fluids with OrientationDependent Bonding: Equilibrium, Stability, and Implications from the Phase Behavior of Supercooled Water," J. Phys. Chem. 99, 3781-3793 (1995).

49. C. F. Tejero and M. Baus, "Liquid Polymorphism of Simple Fluids within a van der Waals Theory," Phys. Rev. E 57, 4821-4823 (1998).

50. G. Franzese and H. E. Stanley, "A theory for discriminating the mechanism responsible for the water density anomaly", Physica A 314, 508 (2002).

51. G. Franzese and H. E. Stanley, "Liquid-liquid critical point in a Hamiltonian model for water: analytic solution", J. Phys.: Cond. Mat. 14, 2193 (2002).

52. G. Franzese, M. I. Marqués, and H. E. Stanley, "Intramolecular coupling as a mechanism for a liquidliquid phase transition", Phys. Rev. E. 67, 011103 (2003).

53. G. Franzese and H. E. Stanley, "The Widom line of supercooled water", J. Phys.: Cond. Mat. 19, 205126 (2007).

54. H. Kanno, R. Speedy, and C. A. Angell, "Supercooling of Water to $-92^{\circ} \mathrm{C}$ under Pressure," Science 189 , 880-881 (1975).

55. O. Mishima, "Reversible first-order transition between two $\mathrm{H}_{2} \mathrm{O}$ amorphs at $-0.2 \mathrm{GPa}$ and $135 \mathrm{~K}$," J. Chem. Phys. 100, 5910-5912 (1994).

56. O. Mishima and H. E. Stanley "Decompression-Induced Melting of Ice IV and the Liquid-Liquid Transition in Water," Nature 392, 164-168 (1998).

57. E. Whalley, D. D. Klug, and Y. P. Handa, "Entropy of Amorphous Ice," Nature 342, 782-783 (1989).

58. G. P. Johari, G. Fleissner, A. Hallbrucker, and E. Mayer, "Thermodynamic Continuity between Glassy and Normal Water," J. Phys. Chem. 98, 4719-4725 (1994).

59. R. J. Speedy, P. G. Debenedetti, R. S. Smith, C. Huang, and B. D. Kay, "The Evaporation Rate, Free Energy, and Entropy of Amorphous Water at 150K," J. Chem. Phys. 105, 240-244 (1996).

60. L. S. Bartell and J. Huang, "Supercooling of Water below the Anomalous Range near $226 \mathrm{~K}, " J$. Phys. Chem. 98, 7455-7457 (1994).

61. P. Brüggeller and E. Mayer, Complete Vitrification in Pure Liquid Water and Dilute Aqueous Solutions," Nature 288, 569-571 (1980).

62. P. W. Bridgman, The Pressure-Volume-Temperature Relations of the Liquid, and the Phase Diagram of Heavy Water," J. Chem. Phys. 3, 597-605 (1935).

63. P. H. Poole, U. Essmann, F. Sciortino, and H. E. Stanley, "Phase Diagram for Amorphous Solid Water," Phys. Rev. E 48, 4605-4610 (1993).

64. H. Tanaka, "Phase Behaviors of Supercooled Water: Reconciling a Critical Point of Amorphous Ices with Spinodal Instability," J. Chem. Phys. 105, 5099-5111 (1996).

65. S. Harrington, R. Zhang, P. H. Poole, F. Sciortino, and H. E. Stanley, "Liquid-Liquid Phase Transition: Evidence from Simulations," Phys. Rev. Lett. 78, 2409-2412 (1997).

66. F. Sciortino, P. H. Poole, U. Essmann, and H. E. Stanley, "Line of Compressibility Maxima in the Phase Diagram of Supercooled Water," Phys. Rev. E 55, 727-737 (1997).

67. S. Harrington, P. H. Poole, F. Sciortino, and H. E. Stanley, "Equation of State of Supercooled SPC/E Water," J. Chem. Phys. 107, 7443-7450 (1997).

68. W. L. Jorgensen, J. Chandrasekhar, J. Madura, R. W. Impey, and M. Klein, "Comparison of Simple Potential Functions for Simulating Liquid Water," J. Chem. Phys. 79, 926 (1983).

69. M. Yamada, S. Mossa, H. E. Stanley, F. Sciortino, "Interplay Between Time-Temperature-Transformation and the Liquid-Liquid Phase Transition in Water," Phys. Rev. Lett. 88, 195701 (2002).

70. D. Paschek, "How the Liquid-Liquid Transition Affects Hydrophobic Hydration in Deeply Supercooled Water," Phys. Rev. Lett. 94, 217802 (2005).

71. E. Shiratani and M. Sasai, "Molecular Scale Precursor of the Liquid-Liquid Phase Transition of Water," J. Chem. Phys. 108, 3264-3276 (1998).

72. M.-C. Bellissent-Funel, "Is There a Liquid-Liquid Phase Transition in Supercooled Water?" Europhys. Lett. 42, 161-166 (1998).

73. H. E. Stanley, S. V. Buldyrev, M. Canpolat, O. Mishima, M. R. Sadr-Lahijany, A. Scala, and F. W. Starr, "The Puzzling Behavior of Water at Very Low Temperature" [opening paper in Proc. International Meeting on Metastable Fluids, Bunsengesellschaft] Physical Chemistry and Chemical Physics (PCCP) 2, 1551-1558 (2000).

74. A. K. Soper and M. A. Ricei, "Structures of HighDensity and Low-Density Water", Phys. Rev. Lett. 84, 2881 (2000) and references cited therein.

75. A. C. Mitus, A. Z. Patashinskii, and B. I. Shumilo, "The Liquid-Liquid Phase Transition," Phys. Lett. 113A, 41-44 (1985).

76. A. C. Mitus and A. Z. Patashinskii, "The Liquid-Liquid Phase Transition. 1. Statistical-Mechanics Description," Acta Physica Polonica A 74, 779-796 (1988).

77. R. Zangi and A. E. Mark, "Bilayer Ice and Alternate Liquid Phases of Confined Water," J. Chem. Phys. 119, 1694-1700 (2003).

78. R. Zangi, "Water Confined to a Slab Geometry: A Review of Recent Computer Simulation Studies," J. Phys.-Cond. Mat. 16, S5371-S5388 (2004).

79. P. M. Wiggins, "Role of Water in Some Biological Processes," Microbiological Reviews 54, 432-449 (1990).

80. P. M. Wiggins, "High and Low-Density Water in Gels," Prog. Polym. Sci. 20, 1121-1163 (1995).

81. M.-C. Bellissent-Funel, J.-M. Zanotti, and S. H. Chen, "Slow Dynamics of Water Molecules on the Surface of a Globular Protein," Faraday Discuss. 103, 281-294 (1996).

82. V. Crupi, S. Magazu, D. Majolino, P. Migliardo, V. Venuti, and M.-C. Bellissent-Funel, "Confinement Influence in Liquid Water Studied by Raman and Neutron Scattering," J. Phys. Cond. Matter 12, 3625-3630 (2000);

83. M.-C. Bellissent-Funel, "Hydration in Protein Dynamics and Function," J. Mol. Liq. 84, 39-52 (2000).

84. M.-C. Bellissent-Funel, S. H. Chen, and J. M. Zanotti, "Single-Particle Dynamics of Water-Molecules in Confined Space," Phys. Rev. E 51, $4558-4569$ (1995).

85. M.-C. Bellissent-Fune1, J. Teixeira, K. F. Bradley, and S. 
H. Chen, "Dynamics of Hydration Water in Protein," $J$. de Physique I 2, 995-1001 (1992).

86. S. H. Chen, P. Gallo, and M.-C. Bellissent-Funel, "Slow Dynamics of Interfacial Water," Canadian J. Phys. 73, 703-709 (1995).

87. Z. Dohnálek, G. A. Kimmel, R. L. Ciolli, K. P. Stevenson, R. S. Smith, and B. D. Kay, "The Effect of the Underlying Substrate on the Crystallization Kinetics of Dense Amorphous Solid Water Films," J. Chem. Phys. 112, 5932-5941 (2000).

88. T. M. Truskett, P. G. Debenedetti, and S. Torquato, "Thermodynamic Implications of Confinement for a Waterlike Fluid," J. Chem. Phys. 114, 2401-2418 (2001).

89. J.-M. Zanotti, M.-C. Bellissent-Funel, and S.-H. Chen, "Experimental Evidence of a Liquid-Liquid Transition in Interfacial Water," Europhys. Lett. 71, 91-97 (2005).

90. L. Liu, S.-H. Chen, A. Faraone, C.-W. Yen, and C. Y. Mou, "Pressure Dependence of Fragile-to-Strong Transition and a Possible Second Critical Point in Supercooled Confined Water," Phys. Rev. Lett. 95, $117802(2005)$.

91. M. E. Green and J. Lu, "Monte-Carlo Simulation of the Effects of Charges on Water and Ions in a Tapered Pore," J. Coll. Int. Sci. 171, 117-126 (1995)

92. K. Koga, X. C. Zeng, and H. Tanaka, "Freezing of Confined Water: A Bilayer Ice Phase in Hydrophobic Nanopores," Phys. Rev. Lett. 79, 5262-5265 (1997).

93. J. Slovak, K. Koga, H. Tanaka, and X. C. Zeng, "Confined Water in Hydrophobic Nanopores: Dynamics of Freezing into Bilayer Ice," Phys. Rev. E 60, $5833-5840$ (1999).

94. K. Koga, X. C. Zeng, and H. Tanaka, "Effects of Confinement on the Phase Behavior of Supercooled Water," Chem. Phys. Lett. 285, 278-283 (1998).

95. K. Koga, H. Tanaka, and X. C. Zeng, "First-Order Transition in Confined Water between High-Density Liquid and Low-Density Amorphous Phases," to appear in Nature.

96. R. Bergman and J. Swenson, "Dynamics of Supercooled Water in Confined Geometry," Nature 403, 283-286 (2000).

97. J. Teixeira, J. M. Zanotti, M.-C. Bellissent-Funel, and S. H. Chen, "Water in Confined Geometries," Physica B 234, 370-374 (1997).

98. P. Gallo, "Single Particle Slow Dynamics of Confined Water," Phys. Chem. Phys. 2, 1607-1611 (2000).

99. P. Gallo, M. Rovere, M. A. Ricci, C. Hartnig, and E. Spohr, "Non-Exponential Kinetic Behavior of Confined Water," Europhys. Lett. 49, 183-188 (2000).

100. P. Gallo, M. Rovere, M. A. Ricci, C. Hartnig, and E. Spohr, "Evidence of Glassy Behavior of Water Molecules in Confined States," Philos. Mag. B 79, 1923-1930 (1999).

101. M. Rovere, M. A. Ricci, D. Vellati, and F. Bruni, "A Molecular Dynamics Simulation of Water Confined in a Cylindrical $\mathrm{SiO}_{2}$ Pore," J. Chem. Phys. 108, 9859-9867 (1998).

102. M.-C. Bellissent-Funel, R. Sridi-Dorbez, and L. Bosio, "X-Ray and Neutron Scattering Studies of the Structure of Water at a Hydrophobic Surface," J. Chem. Phys. 104, 10023-10029 (1996).

103. J. Forsman, B. Jonsson, and C. E. Woodward, "Computer
Simulations of Water between Hydrophobic Surfaces: The Hydrophobic Force," J. Phys. Chem-US 100, 15005-15010 (1996).

104. M. Meyer and H. E. Stanley, "Liquid-Liquid Phase Transition in Confined Water: A Monte Carlo Study," $J$. Phys. Chem. B 103, 9728-9730 (1999).

105. P. A. Netz and T. Dorfmuller, "Computer Simulation Studies on the Polymer-Induced Modification of Water Properties in Polyacrylamide Hydrogels," J. Phys. Chem. B 102, 4875-4886 (1998).

106. J.-M. Zanotti, M.-C. Bellissent-Funel, and S. H. Chen, "Relaxational Dynamics of Supercooled Water in Porous Glass," Phys. Rev. E 59, 3084-3093 (1999).

107. M.-C. Bellissent-Funel and J. Teixeira, "Structural and Dynamic Properties of Bulk and Confined Water," in Freeze-Drying/Lyophilization of Pharmaceutical and Biological Products, edited by L. Rey and J. C. May (Marcel Dekker, New York, 1999), Chapter 3, pp. 53-77.

108. H. Tanaka and I. Ohmine, "Large Local Energy Fluctuations in Water," J. Chem. Phys. 87, 6128-6139 (1987).

109. I. Ohmine, H. Tanaka, and P.. G. Wolynes, "Large Local Energy Fluctuations in Water. II. Cooperative Motions and Fluctuations," J. Chem. Phys. 89, 5852-5860 (1988).

110. H. Tanaka and I. Ohmine, "Potential Energy Surfaces for Water Dynamics: Reaction Coordinates, Transition States, and Normal Mode Analyses," J. Chem. Phys. 91 , 6318-6327 (1989).

111. I. Ohmine and H. Tanaka, "Potential Energy Surfaces for Water Dynamics. II. Vibrational Mode Excitations, Mixing, and Relaxations," J. Chem. Phys. 93, 8138-8147 (1990).

112. I. Ohmine and H. Tanaka, "Dynamics of Liquid Water: Fluctuations and Collective Motions," in Molecular Dynamics Simulations, edited by F. Yonezawa (Springer Verlag, Berlin, 1991), pp. 130-138.

113. I. Okabe, H. Tanaka, and K. Nakanishi, "Structure and Phase Transitions of Amorphous Ices," Phys. Rev. E 53 , 2638-2647 (1996).

114. H. Tanaka, "Phase Diagram for Supercooled Water and Liquid-Liquid Transition," in ACS Symposium Series on Experimental and Theoretical Approaches to Supercooled Liquids, edited by J. Fourkas (ACS, 1997), Chap. 18, pp. 233-245.

115. T. Kabeya, Y. Tamai, and H. Tanaka, "Structure and Potential Surface of Liquid Methanol in Low Temperature: A Comparison of Hydrogen Bond Network in Methanol with Water," J. Phys. Chem. B 102, 899-905 (1998).

116. Y. Tamai and H. Tanaka, "Effects of Chain on Structure and Dynamics of Supercooled Water in Hydrogel," Phys. Rev. E 59, 5647-5654 (1999).

117. H. Tanaka, R. Yamamoto, K. Koga, and X. C. Zeng, "Can Thin Disk-Like Clusters Be More Stable Than Compact Droplet-Like Clusters?" Chem. Phys. Lett. 304, 378-384 (1999).

118. J. Slovak, K. Koga, H. Tanaka, and X. C. Zeng, "Confined Water in Hydrophobic Nanopores: Dynamics of Freezing into Bilayer Ice," Phys. Rev. E 60, 5833-5840 (1999).

119. G. T. Gao and X. C. Zeng, and H. Tanaka, "The Melting Temperature of Proton-Disordered Hexagonal Ice: A Computer Simulation of TIP4P Model of Water," J. 
Chem. Phys. 112, 8534-8538(2000).

120. M.-C. Bellissent-Funel, "Status of Experiments Probing the Dynamics of Water in Confinement," Eur. Phys. J. E 12, 83-92 (2003).

121. M.-C. Bellissent-Funel, "Hydrophilic-Hydrophobic Interplay: From Model Systems to Living Systems," C. R. Geoscience 337, 173-179 (2005).

122. P. C. Hemmer and G. Stell, "Fluids with Several Phase Transitions," Phys. Rev. Lett. 24, 1284-1287 (1970).

123. M. Canpolat, F. W. Starr, M. R. Sadr-Lahijany, A. Scala, O. Mishima, S. Havlin and H. E. Stanley, "Local Structural Heterogeneities in Liquid Water under Pressure," Chem. Phys. Lett. 294, 9-12 (1998).

124. S. Sastry, F. Sciortino, and H. E. Stanley, "Limits of Stability of the Liquid Phase in a Lattice Model with Water-Like Properties," J. Chem. Phys. 98, 9863-9872 (1993).

125. C. J. Roberts, A. Z. Panagiotopulos, and P. G. Debenedetti, "Liquid-Liquid Immiscibility in Pure Fluids: Polyamorphism in Simulations of a NetworkForming Fluid," Phys. Rev. Lett. 77, 4386-4389 (1996).

126. E. Shiratani and M. Sasai, "Growth and Collapse of Structural Patterns in the Hydrogen Bond Network in Liquid Water," J. Chem. Phys. 104, 7671-7680 (1996).

127. H. Tanaka, "Fluctuation of Local Order and Connectivity of Water Molecules in Two Phases of Supercooled Water," Phys. Rev. Lett. 80, 113-116 (1998).

128. C. A. Angell, J. Shuppert, and J. C. Tucker, "Anomalous Properties of Supercooled Water: Heat Capacity, Expansivity, and Proton Magnetic Resonance Chemical Shift from 0 to -38 C," J. Phys. Chem. 77, 3092-3099 (1973).

129. F. Sciortino, L. Fabbian, S.-H. Chen, and P. Tartaglia, "Supercooled Water and the Kinetic Glass Transition: 2. Collective Dynamics," Phys. Rev. E 56, 5397-5404 (1997).

130. F. Sciortino, P. Gallo, P. Tartaglia, and S.-H. Chen, "Supercooled Water and the Glass Transition," Phys. Rev. E 54, 6331-6343 (1996).

131. P. Kumar, G. Franzese, S. V. Buldyrev, H. E. Stanley, "Molecular Dynamics Study of Orientational Cooperativity in Water", Phys. Rev. E 73, 041505 (2006).

132. Y. Xie, K. F. Ludwig, G. Morales, D. E. Hare, and C. M. Sorensen, "Noncritical Behavior of Density Fluctuations in Supercooled Water," Phys. Rev. Lett. 71, 2051-2053 (1993).

133. F. Sciortino, P. Poole, H.E.Stanley and S. Havlin, "Lifetime of the Hydrogen Bond Network and Gel-Like Anomalies in Supercooled Water," Phys. Rev. Lett. 64, 1686-1689 (1990).

134. A. Luzar and D. Chandler, "Hydrogen-Bond Kinetics in Liquid Water," Nature 379, 55-57 (1996); A. Luzar and D. Chandler, "Effect of Environment on Hydrogen Bond Dynamics in Liquid Water," Phys. Rev. Lett. 76, 928-931 (1996).

135. F. W. Starr, J. K. Nielsen, and H. E. Stanley. "Fast and Slow Dynamics of Hydrogen Bonds in Liquid Water," Phys. Rev. Lett. 82, 2294-2297 (1999); F. W. Starr, J. K. Nielsen, and H. E. Stanley, "Hydrogen Bond Dynamics for the Extended Simple Point Charge Model of Water," Phys. Rev. E 62, 579-587 (2000).
136. J. R. Errington and P. G. Debenedetti, "Relationship between Structural Order and the Anomalies of Liquid Water," Nature 409, 318-321 (2001).

137. J. M. Kincaid, G. Stell and C. K. Hall, "Isostructural Phase Transitions Due to Core Collapse: I. A OneDimensional Model," J. Chem. Phys. 65, 2161 (1976).

138. E. A. Jagla, "A Model for the Fragile-to-Strong Transition in Water," J. Phys. Cond. Mat. 11, 1025110258 (1999).

139. E. A. Jagla, "Low-Temperature Behavior of CoreSoftened Models: Water and Silica Behavior," Phys. Rev. E 63, 061509 (2001).

140. M. R. Sadr-Lahijany, A. Scala, S. V. Buldyrev and H. E. Stanley, "Liquid State Anomalies for the StellHemmer Core-Softened Potential," Phys. Rev. Lett. 81, 4895-4898 (1998).

141. A. Scala, M. R. Sadr-Lahijany, N. Giovambattista, S. V. Buldyrev, and H. E. Stanley, "Water-Like Anomalies for Core-Softened Models of Fluids: Two Dimensional Systems," Phys. Rev. E 63041202 (2001).

142. A. Scala, M. Reza Sadr-Lahijany, N. Giovambattista, S. V. Buldyrev, and H. E. Stanley, "Applications of the Stell-Hemmer Potential to Understanding Second Critical Points in Real Systems," [Festschrift for G. S. Stell] J. Stat. Phys. 100, 97-106 (2000).

143. G. Franzese, G. Malescio, A. Skibinsky, S. V. Buldyrev, and H. E. Stanley, "Generic Mechanism for Generating a Liquid-Liquid Phase Transition," Nature 409, 692-695 (2001).

144. G. Franzese, G. Malescio, A. Skibinsky, S. V. Buldyrev, and H. E. Stanley, "Metastable Liquid-Liquid Phase Transition in a Single-Component System with only one Crystal Phase and No Density Anomaly," Phys. Rev. E 66, 051206 (2002).

145. A. Skibinsky, S.V. Buldyrev, G. Franzese, G. Malescio, and H. E. Stanley, "Liquid-liquid phase transitions for soft-core attractive potentials", Phys. Rev. E 69, 061206 (2004).

146. G. Malescio, G. Franzese, A. Skibinsky, S. V. Buldyrev, and H. E. Stanley, "Liquid-liquid phase transition for an attractive isotropic potential with wide repulsive range", Phys. Rev. E 71, 061504 (2005).

147. G. Franzese, "Differences between discontinuous and continuous soft-core attractive potentials: the appearance of density anomaly", J. Mol. Liq. 136, 267 (2007).

148. P. Kumar, G. Franzese, and H. E. Stanley, "Predictions of Dynamic Behavior Under Pressure for Two Scenarios to Explain Water Anomalies" (submitted), condmat $/ 0702108$.

149. G. D'Arrigo et al., J. Chem. Phys. 75, 4264 (1981); C. A. Angell and V. Rodgers ibid. 80, 6245 (1984).

150. E. Schwegler et al., Phys. Rev. Lett. 84, 2429 (2000); P. Raiteri et al., ibid. 93, 087801 (2004) and references cited therein.

151. P. Kumar, S. V. Buldyrev, F. Sciortino, E. Zaccarelli, and H. E. Stanley, "Static and Dynamic Anomalies in a Repulsive Spherical Ramp Liquid: Theory and Simulation," Phys. Rev. E 72, 021501 (2005).

152. P. G. Debenedetti, V. S. Raghavan, and S. S. Borick, "Spinodal Curve of some Supercooled Liquids," J. Phys. Chem. 95, 4540-4551 (1991).

153. V. B. Henriques and M. C. Barbosa, "Liquid Polymorphism and Density Anomaly in a Lattice 
Gas Model," Phys. Rev. E 71, 031504 (2005).

154. B. Guillot and Y. Guissani, "Polyamorphism in Low Temperature Water: A Simulation Study," J. Chem. Phys. 119, 11740-11752 (2003).

155. V. B. Henriques, N. Guisoni, M. A. Barbosa, M. Thielo, and M. C. Barbosa, "Liquid Polyamorphism and Double Criticality in a Lattice Gas Model," Molec. Phys. 103, 3001-3007 (2005).

156. A. Barros de Oliveira, G. Franzese, P. A. Netz, and M. C. Barbosa, "Water-like hierarchy of anomalies in a continuous spherical shouldered potential", J. Chem. Phys. (in press), arXiv:0706.2838.

157. E. A. Jagla, "Core-Softened Potentials and the Anomalous Properties of Water," J. Chem. Phys. 111, 8980-8986 (1999).

158. T. H. Hall, L. Merril, and J. D. Barnett, "High Pressure Polymorphism in Cesium," Science 146, 1297-1299 (1964).

159. A. Scala, F. W. Starr, E. La Nave, F. Sciortino and H. E. Stanley, "Configurational Entropy and Diffusivity of Supercooled Water," Nature 406, 166-169 (2000).

160. Z. Yan, S. V. Buldyrev, N. Giovambattista, and H. E. Stanley "Structural Order for One-Scale and Two-Scale Potentials," Phys. Rev. Lett. 95, 130604 (2005).

161. Z. Yan, S. V. Buldyrev, N. Giovambattista, P. G. Debenedetti, and H. E. Stanley, "Family of Tunable Spherically-Symmetric Potentials that Span the Range from Hard Spheres to Water-like Behavior," Phys. Rev. E 73, 051204 (2006)

162. Z. Yan, S. V. Buldyrev, P. Kumar, N. Giovambattista, P. G. Debenedetti, and H. E. Stanley, "Structure of the First- and Second-Neighbor Shells of Simulated Water: Quantitative Relation to Translational and Orientational Order," Phys. Rev. E 76, 051201 (2007).

163. N. Giovambattista, P. J. Rossky, and P. G. Debenedetti, "Effect of Pressure on the Phase Behavior and Structure of Water Confined between Nanoscale Hydrophobic and Hydrophilic Plates," Phys. Rev. E 73, 041604 (2006).

164. C. A. Ange11, "Amorphous Water," Ann. Rev. Phys. Chem. 55, 559-583 (2004).

165. P. H. Poole, T. Grande, C. A. Angell, and P. F. McMillan, "Polymorphic Phase Transitions in Liquids and Glasses," Science 275, 322-323 (1997).

166. J. L. Yarger, and G. H. Wolf, "Chemistry: Polymorphism in Liquids," Science 306, 820-821 (2004)

167. F. Mallamace, M. Broccio, C. Corsaro, A. Faraone, U. Wanderlingh, L. Liu, C.-Y. Mou, and S.-H. Chen, "The Fragile-to-Strong Dynamic Crossover Transition in Confined Water: NMR Results," J. Chem. Phys. 124, $161102(2006)$

168. L. Xu, P. Kumar, S. V. Buldyrev, S.-H. Chen, P. H. Poole, F. Sciortino, and H. E. Stanley, "Relation between the Widom Line and the Dynamic Crossover in Systems with a Liquid-Liquid Critical Point," Proc. Natl. Acad. Sci. 102, 16558-16562 (2005).

169. A. Faraone, L. Liu, C.-Y. Mou, C.-W. Yen, and S.-H. Chen, "Fragile-to-Strong Liquid Transition in Deeply Supercooled Confined Water," J. Chem. Phys. 121, 10843-10846 (2004)

170. L. Liu, "Study of Slow Dynamics in Supercooled Water by Molecular Dynamics and Quasi-Elastic Neutron Scattering," Ph.D. thesis, M.I.T., September 2005.

171. J. M. H. Levelt, Measurements of the Compressibility of
Argon in the Gaseous and Liquid Phase, Ph.D. Thesis (University of Amsterdam, Van Gorkum and Co., 1958).

172. M. A. Anisimov, J. V. Sengers, and J. M. H. LeveltSengers, "Near-Critical Behavior of Aqueous Systems," in Aqueous System at Elevated Temperatures and Pressures: Physical Chemistry in Water, Stream and Hydrothermal Solutions, edited by D. A. Palmer, R. Fernandez-Prini, and A. H. Harvey (Elsevier, Amsterdam, 2004).

173. M.-C. Bellissent-Fune1, ed., Hydration Processes in Biology: Theoretical and Experimental Approaches [Proc. NATO Advanced Study Institutes, Vol. 305] (IOS Press, Amsterdam, 1999).

174. G. W. Robinson, S.-B. Zhu, S. Singh, and M. W. Evans, Water in Biology, Chemistry, and Physics: Experimental Overviews and Computational Methodologies (World Scientific, Singapore, 1996).

175. C. A. Angell, R. D. Bressel, M. Hemmatti, E. J. Sare, and J. C. Tucker, "Water and Its Anomalies in Perspective: Tetrahedral Liquids With and Without Liquid-Liquid Phase Transitions," Phys. Chem. Chem. Phys. 2, 1559-1566 (2000).

176. P. G. Debenedetti, "Supercooled and Glassy Water," J. Phys.: Condens. Matter 15, R1669-R1726 (2003).

177. C. A. Angell, "Water-II is a Strong Liquid," J. Phys. Chem. 97, 6339-6341 (1993).

178. F. W. Starr, C. A. Angell, and H. E. Stanley, "Prediction of Entropy and Dynamic Properties of Water below the Homogeneous Nucleation Temperature," Physica A $\mathbf{3 2 3}$, 51-66 (2003).

179. J. Horbach and W. Kob, "Static and Dynamic Properties of a Viscous Silica Melt," Phys. Rev. B 60, 3169-3181 (1999).

180. E. W. Lang and H. D. Lüdemann, "Anomalies of Liquid Water" Angew Chem. Intl. Ed. Engl. 21, 315-329 (1982).

181. F. X. Prielmeier, E. W. Lang, R. J. Speedy, H. D. Lüdemann, "Diffusion in Supercooled Water to 300-MPA," Phys. Rev. Lett. 59, 1128-1131 (1987).

182. K. Ito, C. T. Moynihan, and C. A. Angell, "Thermodynamic Determination of Fragility in Liquids and a Fragile-to-Strong Liquid Transition in Water," Nature 398, 492-495 (1999).

183. H. Tanaka, "A New Scenario of the Apparent Fragileto-Strong Transition in Tetrahedral Liquids: Water as an Example," J. Phys.: Condens. Matter 15, L703-L711 (2003).

184. J. Swenson, H. Jansson, W. S. Howells, and S. Longeville, "Dynamics of Water in a Molecular Sieve by Quasielastic Neutron Scattering," J. Chem. Phys. 122, 084505 (2005).

185. S. Sastry and C. A. Angell, "Liquid-Liquid Phase Transition in Supercooled Silicon," Nature Materials 2 , 739-743 (2003)

186. I. Saika-Voivod, P. H. Poole, and F. Sciortino, "Fragileto-Strong Transition and Polyamorphism in the Energy Landscape of Liquid Silica," Nature 412, 514-517 (2001).

187. D. C. Rapaport, in The Art of Molecular Dynamics Simulation (Cambridge University Press, Cambridge, 1995).

188. F. H. Stillinger and A. Rahman, "Molecular Dynamics Study of Temperature Effects on Water Structure and 
Kinetics," J. Chem. Phys. 57, 1281-1292 (1972).

189. W. Götze and L. Sjögren, "Relaxation Processes in Supercooled Liquids," Rep. Prog. Phys. 55, 241-376 (1992).

190. P. H. Poole, I. Saika-Voivod, and F. Sciortino, "Density Minimum and Liquid-Liquid Phase Transition," J. Phys.: Condens. Matter 17, L431-L437 (2005).

191. N. Jakse, L. Hennet, D. L. Price, S. Krishnan, T. Key, E. Artacho, B. Glorieux, A. Pasture1, and M.-L. Saboungi, "Structural Changes on Supercooling Liquid Silicon," Appl. Phys. Lett. 83, 47344736 (2003).

192. L. Xu, F. Mallamace, F. W. Starr, Z. Yan, S. V. Buldyrev, and H. E. Stanley, "Interpretation for the Breakdown of Stokes-Einstein Relation in Water" (preprint).

193. P. Kumar, S. V. Buldyrev, S. L. Becker, P. H. Poole, F. W. Starr, and H. E. Stanley, "Relation between the Widom line and the Breakdown of the Stokes-Einstein Relation in Supercooled Water" Proc. Natl. Acad. Sci. 104, 9575-9579 (2007).

194. P. Kumar, S. V. Buldyrev, and H. E. Stanley "Space-Time Correlations in the Orientational Order Parameter and the Orientational Entropy of Water" (submitted).

195. S.-H. Chen, F. Mallamace, C.-Y. Mou, M. Broccio, C. Corsaro, and A. Faraone, "The Violation of the Stokes-Einstein Relation in Supercooled Water," Proc. Nat. Acad. Sciences USA 103, 12974-12978 (2006)

196. M. G. Mazza, N. Giovambattista, F. W. Starr, and H. E. Stanley, "Relation between Rotational and Translational Dynamic Heterogeneities in Water," Phys. Rev. Lett. 96, 057803 (2006).

197. M. G. Mazza, N. Giovambattista, H. E. Stanley, and F. W. Starr, "Connection of Translational and Rotational Dynamical Heterogeneities with the Breakdown of the Stokes-Einstein and Stokes-Einstein-Debye Relations in Water," Phys. Rev. E 76, 031202 (2007).

198. S.-H. Chen, L. Liu, E. Fratini, P. Baglioni, A. Faraone, and E. Mamontov, "The Observation of Fragile-to-strong Dynamic Crossover in Protein Hydration Water," Proc. Natl. Acad. Sci. USA 103, 9012 (2006).

199. F. Mallamace, S.-H. Chen, M. Broccio, C. Corsaro, V. Crupi, D. Majolino, V. Venuti, P. Baglioni, E. Fratini, C. Vannucci, and H. E. Stanley, "The Role of the Solvent in the Dynamical Transitions of Proteins: The Case of the Lysozyme-Water System," J. Chem. Phys. 127, 045104 (2007); F. Mallamace, C. Branca, M. Broccio, C. Corsaro, N. Gonzalez-Segredo, H. E. Stanley, and S.-H. Chen, "Transport Properties of Supercooled Confined Water," Euro. Phys. J. xx, xx (2008).

200. P. Kumar, F. W. Starr, S. V. Buldyrev, and H. E. Stanley, "Effect of Water-Wall Iteraction Potential on the properties of Nanoconfined Water," Phys. Rev. E 75, 011202 (2007).

201. P. Kumar, S. V. Buldyrev, and H. E. Stanley, "Dynamic Crossover and Liquid-Liquid Critical Point in the TIP5P Model of Water," in Soft Matter under Extreme Pressures: Fundamentals and Emerging Technologies, edited by S. J. Rzoska and V. Mazur [Proc. NATO ARW, Odessa, Oct. 2005] (Springer, Berlin, 2006).

202. D. Liu, Y. Zhang, C.-C. Chen, C.-Y. Mou, P. H. Poole, and S.-H. Chen, "Observation of the Density Minimum in Deeply Supercooled Confined Water," Proc. Natl. Acad. Sci. 104, 9570-9574 (2007).

203. D. A. Fuentevilla and M. A. Anisimov, "Scaled Equation of State for Supercooled Wataer near the Liquid-Liquid Critical Point," Phys. Rev. Lett. 97, 195702 (2006).

204. T. Morishita, "How Does Tetrahedral Structure Grow in Liquid Silicon on Supercooling?" Phys. Rev. Lett. 97 (2006) 165502.

205. L. Xu, S. V. Buldyrev, C. A. Angell, and H. E. Stanley, "Thermodynamics and Dynamics of the TwoScale Spherically Symmetric Jagla Ramp Model of Anomalous Liquids," Phys. Rev. E 74031108 (2006).

206. F. Mallamace, C. Branca, M. Broceio, C. Corsaro, C.-Y. Mou, and S.-H. Chen, "The Anomalous Behavior of the Density of Water in the Range $30 \mathrm{~K}<T<373 \mathrm{~K}$," C. Y. Mou, and S.-H. Chen, Proc. Natl. Acad. Sci. USA 104, 18387-18391 (2007).

207. F. Mallamace, C. Corsaro, M. Broccio, C. Branca, N. González-Segredo, J. Spooren, S.-H. Chen, and H. E. Stanley, "NMR Evidence of a Sharp Change in a Measure of Local Order in Deeply Supercooled Water" (in press).

208. S.-H. Chen, F. Mallamace, L. Liu, D. Z. Liu, X. Q. Chu, Y. Zhang, C. Kim. A. Faraone, C.-Y. Mou, E. Fratini, P. Baglioni, A. I. Kolesnikov, and V. GarciaSakai, "Dynamic Crossover Phenomenon in Confined Supercooled water and its relation to the existence of a liquid-liquid critical point in water," AIP Conf Proc. (in press).

209. S. Maruyama, K. Wakabayashi, and M. Oguni, AIP Conf. Proc. 708, 675-676 (2004).

210. U. Raviv, P. Laurat, and J. Klein, Fluidity of water confined to subnanometre films, Nature 413 (2001) $51-54$.

211. P. Kumar, Z. Yan, L. Xu, M. G. Mazza, S. V. Buldyrev, S.-H. Chen. S. Sastry, and H. E. Stanley, "Glass Transition in Biomolecules and the Liquid-Liquid Critical Point of Water," Phys. Rev. Lett. 97, 177802 (2006).

212. J. M. Zanotti, M.-C. Bellissent-Funel, and J. Parrello, Biophys. J. 76, 2390 (1999)

213. D. Ringe, G. A. Petsko, Biophys. Chem. 105, 667 (2003).

214. J. Wang, P. Cieplak and P. A. Kollman, J. Comp. Chem. 21, 1049 (2000); E. J. Sorin and V. S. Pande, Biophys. J. 88, 2472 (2005).

215. B. F. Rasmussen, M. Ringe, and G. A. Petsko, Nature 357, 423 (1992).

216. D. Vitkup, D. Ringe, G. A. Petsko, and M. Karplus, Nat. Struct. Biol. 7, 34 (2000).

217. M. Yamada, S. Mossa, H. E. Stanley, F. Sciortino, Phys. Rev. Lett. 88, 195701 (2002).

218. A. P. Sokolov, H. Grimm, A. Kisliuk and A. J. Dianoux, J. Chem. Phys. 110, 7053 (1999).

219. W. Doster, S. Cusack, and W. Petry, Nature 338, 754 (1989).

220. J. Norberg and L. Nilsson, Proc. Natl. Acad. Sci. USA 93, 10173 (1996).

221. M. Tarek and D. J. Tobias, Phys. Rev. Lett. 88, 138101 (2002); Biophys. J. 79, 3244 (2000).

222. H. Hartmann, F. Parak, W. Steigemann, G. A. Petsko, D. R. Ponzi, H. Frauenfelder, Proc. Natl. Acad. Sci. USA 79, 4067 (1982).

223. A. L. Tournier, J. Xu, and J. C. Smith, Biophys. J. 85, 1871 (2003).

224. A. L. Lee and A. J. Wand, Nature 411501 (2001). 
225. E. Lindahl, B. Hess, and D. van der Spoel, J. Molecular Modeling 7, 306 (2001).

226. P. J. Artymiuk, C. C. F. Blake, D. W. Rice, K. S. Wilson, Acta Crystallogr. B 38, 778 (1982).

227. H. R. Drew, R. M. Wing, T. Takano, C. Broka, S. Tanaka, K. Itakura and R. E. Dickerson, Proc. Natl. Acad. Sci. USA 78, 2179 (1981).

228. S.-H. Chen, L. Liu, X. Chu, Y. Zhang, E. Frattini, P. Baglioni, A. Faraone, and E. Mamontov, J. Chem. Phys. 125,171103 (2006). 